Supporting Information

\title{
Convenient Synthesis of Highly Functionalized Pyrazolines via Mild, Photoactivated 1,3-Dipolar Cycloaddition
}

\author{
Yizhong Wang, Claudia Rivera Vera, and Qing Lin* \\ Department of Chemistry, University at Buffalo, The State University of New York \\ Buffalo, New York 14260-3000
}

\section{General Information}

All photo reactions were carried out under the ambient condition using oven-dried quartz test tubes with magnetic stirring. Solvents and chemicals were purchased from commercial sources and used directly without further purification. Flash chromatography was performed using SiliCycle P60 silica gel $(40-63 \mu \mathrm{m}, 60 \AA) . \quad{ }^{1} \mathrm{H}$ NMR spectra were recorded with Inova-300, -400 or $-500 \mathrm{MHz}$ spectrometers, and chemical shifts were reported in ppm using either TMS or deuterated solvents as internal standards (TMS, 0.00; $\mathrm{CDCl}_{3}, 7.26 ; \mathrm{C}_{6} \mathrm{D}_{6}, 7.15 ; \mathrm{DMSO}-\mathrm{d}_{6}, 2.50$ ). Multiplicity was reported as the follows: $\mathrm{s}=$ singlet, $\mathrm{d}=$ doublet, $\mathrm{t}=$ triplet, $\mathrm{q}=$ quartet, $\mathrm{m}=$ multiplet, $\mathrm{b}=$ broad. ${ }^{13} \mathrm{C}$ NMR spectra were recorded at $75.4 \mathrm{MHz}$, and chemical shifts were reported in ppm using the deuterated solvents as internal standards $\left(\mathrm{CDCl}_{3}, 77.0 ; \mathrm{DMSO}-\mathrm{d}_{6}, 39.5 ; \mathrm{C}_{6} \mathrm{D}_{6}, 128.0\right)$.

\section{Experimental Procedures and Characterization Data}

All tetrazoles were synthesized according to a literature procedure. ${ }^{1}$ Yields of tetrazoles were based on the corresponding aldehyde starting materials.

5-(4-methoxyphenyl)-2-phenyl-2H-tetrazole (Table 1; Table 3, entry 1): 1a $60 \%$ yield: ${ }^{1} \mathrm{H}$ NMR $\left(300 \mathrm{MHz}, \mathrm{CDCl}_{3}\right) \delta 8.20(\mathrm{~d}, J=8.4 \mathrm{~Hz}, 4 \mathrm{H}), 7.58(\mathrm{t}, J=$ $6.9 \mathrm{~Hz}, 2 \mathrm{H}), 7.49(\mathrm{t}, J=7.2 \mathrm{~Hz}, 1 \mathrm{H}), 7.04(\mathrm{~d}, J=9 \mathrm{~Hz}, 2 \mathrm{H}) ;{ }^{13} \mathrm{C} \mathrm{NMR}\left(75.4 \mathrm{MHz}, \mathrm{CDCl}_{3}\right): \delta 165.6$, $162.0,137.5,130.1,130.0,129.1,120.3,114.9,55.9$.

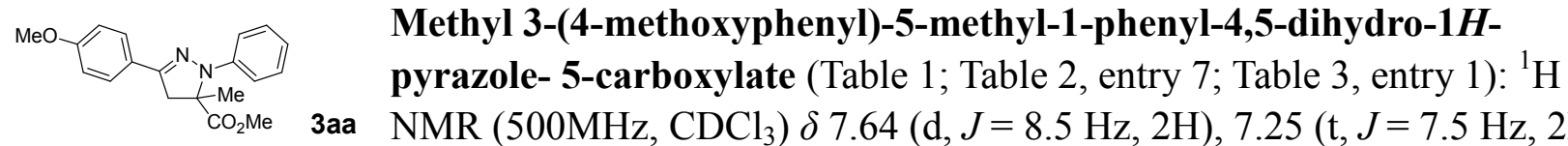

\section{Methyl 3-(4-methoxyphenyl)-5-methyl-1-phenyl-4,5-dihydro-1 $H$ -}

$\mathrm{CO}_{2} \mathrm{Me} \quad$ 3aa $\operatorname{NMR}\left(500 \mathrm{MHz}, \mathrm{CDCl}_{3}\right) \delta 7.64(\mathrm{~d}, J=8.5 \mathrm{~Hz}, 2 \mathrm{H}), 7.25(\mathrm{t}, J=7.5 \mathrm{~Hz}, 2 \mathrm{H})$, $7.09(\mathrm{~d}, J=8.0 \mathrm{~Hz}, 2 \mathrm{H}), 6.92(\mathrm{~d}, J=9.0 \mathrm{~Hz}, 2 \mathrm{H}), 6.86(\mathrm{t}, J=7.5 \mathrm{~Hz}, 1 \mathrm{H}), 3.84(\mathrm{~s}, 3 \mathrm{H}), 3.76(\mathrm{~s}, 3 \mathrm{H})$, $3.66(\mathrm{~d}, J=16.5 \mathrm{~Hz}, 1 \mathrm{H}), 3.25(\mathrm{~d}, J=16.5 \mathrm{~Hz}, 1 \mathrm{H}), 1.63(\mathrm{~s}, 3 \mathrm{H}) ;{ }^{13} \mathrm{C} \mathrm{NMR}\left(75.4 \mathrm{MHz}, \mathrm{CDCl}_{3}\right) \delta$ $174.4,160.2$, 145.1, 143.5, 129.0, 127.2, 125.1, 119.8, 114.5, 114.0, 68.8, 55.3, 52.9, 48.3, 21.0.

\section{General procedure for the photoactivated 1, 3-dipolar cycloaddition (Tables 2 and 3)}

A solution containing tetrazole $1(0.022 \mathrm{mmol})$ and dipolarophile $2(0.022-0.050 \mathrm{mmol})$ was irradiated with a hand-held 302-nm UV lamp for $2 \mathrm{~h}$. The solvent and excess reagent was subsequently removed under the reduced pressure to give a crude product which typically was already very pure for volatile dipolarophiles based on ${ }^{1} \mathrm{H}$ and ${ }^{13} \mathrm{C}$ NMR data. When further 
purification was warranted, the crude product was purified by flash chromatography.

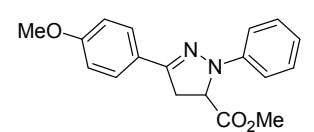

Methyl 3-(4-methoxyphenyl)-1-phenyl-4,5-dihydro-1 $H$-pyrazole-5-

carboxylate (Table 2, entry 1$):{ }^{1} \mathrm{H} \mathrm{NMR}\left(500 \mathrm{MHz}, \mathrm{CDCl}_{3}\right) \delta 7.65(\mathrm{~d}, J=9.0 \mathrm{~Hz}$, $2 \mathrm{H}), 7.28(\mathrm{t}, J=8.0 \mathrm{~Hz}, 2 \mathrm{H}), 7.10(\mathrm{~d}, J=8.5 \mathrm{~Hz}, 2 \mathrm{H}), 6.92(\mathrm{~d}, J=8.5 \mathrm{~Hz}, 2 \mathrm{H})$, $6.86(\mathrm{t}, J=7.5 \mathrm{~Hz}, 1 \mathrm{H}), 4.78(\mathrm{dd}, J=12.5,6.5 \mathrm{~Hz}, 1 \mathrm{H}), 3.84(\mathrm{~s}, 3 \mathrm{H}), 3.75(\mathrm{~s}, 3 \mathrm{H}), 3.65(\mathrm{dd}, J=17.0$, $12.5 \mathrm{~Hz}, 1 \mathrm{H}), 3.39(\mathrm{dd}, J=17.5,7.0 \mathrm{~Hz}, 1 \mathrm{H}) ;{ }^{13} \mathrm{C} \mathrm{NMR}\left(75.4 \mathrm{MHz}, \mathrm{CDCl}_{3}\right) \delta 172.1,160.4,146.9$, $144.9,129.1,127.4,124.8,119.5,114.0,112.9,61.7,55.3,52.7,38.4$.

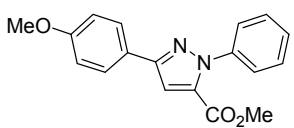

Methyl 3-(4-methoxyphenyl)-1-phenyl-1 $H$-pyrazole-5-carboxylate (Table 2, entry 1, oxidation product): ${ }^{1} \mathrm{H} \mathrm{NMR}\left(400 \mathrm{MHz}, \mathrm{CDCl}_{3}\right) \delta 7.80(\mathrm{~d}, J=8.8 \mathrm{~Hz}$, 2H), 7.49-7.48 (m, 5H), $7.25(\mathrm{~s}, 1 \mathrm{H}), 3.85(\mathrm{~s}, 3 \mathrm{H}), 3.82(\mathrm{~s}, 3 \mathrm{H}) ;{ }^{13} \mathrm{C}$ NMR $(75.4$ $\left.\mathrm{MHz}, \mathrm{CDCl}_{3}\right) \delta 160.4,160.1,151.9,140.9,134.6,129.1,127.6,126.6,125.4,114.7,109.6,55.8$, 52.5; HRMS (ESI) calcd for $\mathrm{C}_{18} \mathrm{H}_{17} \mathrm{~N}_{2} \mathrm{O}_{3} 309.1234\left[\mathrm{M}+\mathrm{H}^{+}\right]$, found 309.1226.

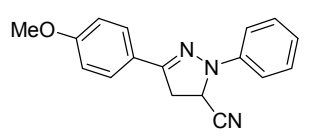

\section{3-(4-methoxyphenyl)-1-phenyl-4,5-dihydro-1 $H$-pyrazole-5-carbonitrile} (Table 2, entry 2): ${ }^{1} \mathrm{H}$ NMR $\left(500 \mathrm{MHz}, \mathrm{CDCl}_{3}\right) \delta 7.65(\mathrm{~d}, J=8.5 \mathrm{~Hz}, 2 \mathrm{H}), 7.36(\mathrm{t}$, $J=9.0 \mathrm{~Hz}, 2 \mathrm{H}), 7.25(\mathrm{~d}, J=8.0 \mathrm{~Hz}, 2 \mathrm{H}), 7.00(\mathrm{t}, J=7.5 \mathrm{~Hz}, 1 \mathrm{H}), 6.94(\mathrm{~d}, J=9.0$ $\mathrm{Hz}, 2 \mathrm{H}), 4.93(\mathrm{dd}, J=9.5,1.5 \mathrm{~Hz}, 1 \mathrm{H}), 3.85(\mathrm{~s}, 3 \mathrm{H}), 3.63(\mathrm{~d}, J=2.5 \mathrm{~Hz}, 1 \mathrm{H}), 3.61(\mathrm{~s}, 1 \mathrm{H}) ;{ }^{13} \mathrm{C} \mathrm{NMR}$ $\left(75.4 \mathrm{MHz}, \mathrm{CDCl}_{3}\right) \delta 160.9,148.2,144.2,129.4,127.6,123.8,121.4,117.3,114.4,114.2,55.4,50.1$, 38.9 .

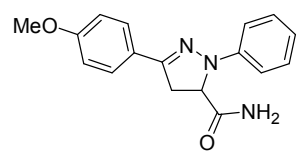

\section{3-(4-methoxyphenyl)-1-phenyl-4,5-dihydro- $1 \mathrm{H}$-pyrazole-5-carboxamide} (Table 2, entry 3): ${ }^{1} \mathrm{H}$ NMR $\left(300 \mathrm{MHz}, \mathrm{DMSO}_{-} \mathrm{d}_{6}\right) \delta 7.75(\mathrm{~s}, 1 \mathrm{H}), 7.68(\mathrm{~d}, J=8.7$ $\mathrm{Hz}, 2 \mathrm{H}), 7.30(\mathrm{~s}, 1 \mathrm{H}), 7.24$ (t, $J=7.8 \mathrm{~Hz}, 2 \mathrm{H}), 7.00(\mathrm{~d}, J=7.2 \mathrm{~Hz}, 4 \mathrm{H}), 6.78$ (t, $J$ $=7.5 \mathrm{~Hz}, 1 \mathrm{H}), 4.58(\mathrm{dd}, J=12.6,7.8 \mathrm{~Hz}, 1 \mathrm{H}), 3.80(\mathrm{~s}, 3 \mathrm{H}), 3.71(\mathrm{dd}, J=17.7,12.6 \mathrm{~Hz}, 1 \mathrm{H}), 3.21$ $(\mathrm{dd}, J=17.4,7.5 \mathrm{~Hz}, 1 \mathrm{H}),{ }^{13} \mathrm{C}$ NMR $\left(75.4 \mathrm{MHz}, \mathrm{DMSO}-\mathrm{d}^{6}\right) \delta 172.9,159.8,147.4,145.0,128.9$, 127.3, 124.6, 118.5, 114.1, 112.3, 62.3, 55.2; HRMS (ESI) calcd for $\mathrm{C}_{17} \mathrm{H}_{18} \mathrm{~N}_{3} \mathrm{O}_{2} 296.1394$ [M + $\mathrm{H}^{+}$], found 296.1385 .

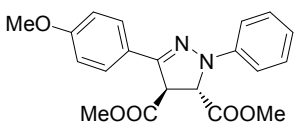

$\left(4 \mathrm{R}^{*}, 5 \mathrm{~S} *\right.$-dimethyl 3-(4-methoxyphenyl)-1-phenyl-4,5-dihydro-1 $H$-pyrazole4,5-dicarboxylate (Table 2, entry 4, racemic form): ${ }^{1} \mathrm{H}$ NMR $\left(500 \mathrm{MHz}, \mathrm{C}_{6} \mathrm{D}_{6}\right) \delta$ $7.82(\mathrm{~d}, J=9.0 \mathrm{~Hz}, 2 \mathrm{H}), 7.20(\mathrm{t}, J=8.0 \mathrm{~Hz}, 2 \mathrm{H}), 7.20(\mathrm{t}, J=8.0 \mathrm{~Hz}, 2 \mathrm{H}), 6.82(\mathrm{t}$, $J=7.5 \mathrm{~Hz}, 1 \mathrm{H}), 6.72(\mathrm{~d}, J=9.0 \mathrm{~Hz}, 2 \mathrm{H}), 5.34(\mathrm{~d}, J=5.0 \mathrm{~Hz}, 1 \mathrm{H}), 4.63(\mathrm{~d}, J=4.5 \mathrm{~Hz}, 1 \mathrm{H}), 3.21(\mathrm{~s}$, $3 \mathrm{H}), 3.09(\mathrm{~s}, 3 \mathrm{H}), 3.04(\mathrm{~s}, 3 \mathrm{H}) ;{ }^{13} \mathrm{C}$ NMR (75.4 MHz, $\left.\mathrm{C}_{6} \mathrm{D}_{6}\right) \delta 170.1,169.2,160.9,144.7,144.1$, $129.5,124.5,120.4,114.4,113.8,66.4,56.3,54.8,52.4,52.2$; HRMS (ESI) calcd for $\mathrm{C}_{20} \mathrm{H}_{21} \mathrm{~N}_{2} \mathrm{O}_{5}$ $369.1445\left[\mathrm{M}+\mathrm{H}^{+}\right]$, found 369.1450 .

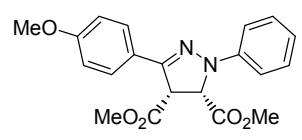

$\left(4 S^{*}, 5 \mathrm{~S} *\right.$-dimethyl 3-(4-methoxyphenyl)-1-phenyl-4,5-dihydro-1 $H$-pyrazole4,5-dicarboxylate (Table 2 , entry 5 , racemic form): ${ }^{1} \mathrm{H}$ NMR $\left(500 \mathrm{MHz}, \mathrm{C}_{6} \mathrm{D}_{6}\right) \delta$ $7.83(\mathrm{~d}, J=8.5 \mathrm{~Hz}, 2 \mathrm{H}), 7.36(\mathrm{~d}, J=8.0 \mathrm{~Hz}, 2 \mathrm{H}), 7.21(\mathrm{t}, J=8.5 \mathrm{~Hz}, 2 \mathrm{H}), 6.82(\mathrm{t}$, $J=7.5 \mathrm{~Hz}, 1 \mathrm{H}), 6.72(\mathrm{~d}, J=9.0 \mathrm{~Hz}, 2 \mathrm{H}), 5.34(\mathrm{~d}, J=4.5 \mathrm{~Hz}, 1 \mathrm{H}), 4.63(\mathrm{~d}, J=5.0 \mathrm{~Hz}, 1 \mathrm{H}), 3.21(\mathrm{~s}$, $3 \mathrm{H}), 3.09(\mathrm{~s}, 3 \mathrm{H}), 3.04(\mathrm{~s}, 3 \mathrm{H}) ;{ }^{13} \mathrm{C}$ NMR $\left(75.4 \mathrm{MHz}, \mathrm{C}_{6} \mathrm{D}_{6}\right) \delta 170.1,169.2,160.9,144.7,144.1$, $129.5,124.5,120.4,114.4,113.8,66.4,56.3,54.8,52.4,52.2 ;$ HRMS (ESI) calcd for $\mathrm{C}_{20} \mathrm{H}_{21} \mathrm{~N}_{2} \mathrm{O}_{5}$ 
369.1445 [M $\left.+\mathrm{H}^{+}\right]$, found 369.1442 .

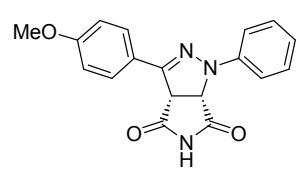

(3aS*,6aS*)-3-(4-methoxyphenyl)-1-phenyl-1,6a-dihydropyrrolo[3,4-c]pyrazole-4,6(3aH,5H)-dione (Table 2, entry 6, racemic form): ${ }^{1} \mathrm{H}$ NMR $(500 \mathrm{MHz}$, DMSO-d $\left._{6}\right) \delta 11.83(\mathrm{~s}, 1 \mathrm{H}), 7.92(\mathrm{~d}, J=9.0 \mathrm{~Hz}, 2 \mathrm{H}), 7.44(\mathrm{~d}, J=8.0 \mathrm{~Hz}, 2 \mathrm{H})$, $7.31(\mathrm{t}, J=7.5 \mathrm{~Hz}, 2 \mathrm{H}), 7.04(\mathrm{~d}, J=9.0 \mathrm{~Hz}, 2 \mathrm{H}), 6.91(\mathrm{t}, J=7.0 \mathrm{~Hz}, 1 \mathrm{H}), 5.25(\mathrm{~d}$, $J=10.5 \mathrm{~Hz}, 1 \mathrm{H}), 5.10(\mathrm{~d}, J=10.5 \mathrm{~Hz}, 1 \mathrm{H}), 3.82(\mathrm{~s}, 3 \mathrm{H}) ;{ }^{13} \mathrm{C}$ NMR $\left(75.4 \mathrm{MHz}, \mathrm{DMSO}-\mathrm{d}_{6}\right) \delta 175.1$, $1173.9,160.2,144.9,144.1,128.9,128.6,123.1,120.2,113.9,113.6,66.6,55.2,55.0$; HRMS (ESI) calcd for $\mathrm{C}_{18} \mathrm{H}_{16} \mathrm{~N}_{3} \mathrm{O}_{3} 322.1186\left[\mathrm{M}+\mathrm{H}^{+}\right]$, found 322.1180 .

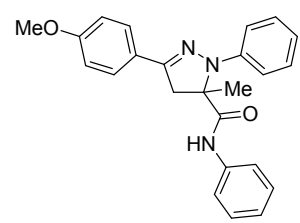

3-(4-methoxyphenyl)-5-methyl-N,1-diphenyl-4,5-dihydro-1 $H$-pyrazole-5carboxamide (Table 2, entry 8): ${ }^{1} \mathrm{H}$ NMR $\left(500 \mathrm{MHz}, \mathrm{CDCl}_{3}\right) \delta 8.52(\mathrm{~s}, 1 \mathrm{H}), 7.69$ $(\mathrm{d}, J=9.0 \mathrm{~Hz}, 2 \mathrm{H}), 7.54(\mathrm{~d}, J=8.5 \mathrm{~Hz}, 2 \mathrm{H}), 7.35-7.23(\mathrm{~m}, 7 \mathrm{H}), 7.13(\mathrm{t}, J=7.0$ $\mathrm{Hz}, 1 \mathrm{H}), 6.99-6.95(\mathrm{~m}, 3 \mathrm{H}), 3.86(\mathrm{~s}, 3 \mathrm{H}), 3.63(\mathrm{~d}, J=17.0 \mathrm{~Hz}, 1 \mathrm{H}), 3.46(\mathrm{~d}, J=$ $17.0 \mathrm{~Hz}, 1 \mathrm{H}), 1.52(\mathrm{~s}, 3 \mathrm{H}) ;{ }^{13} \mathrm{C} \mathrm{NMR}\left(75.4 \mathrm{MHz}, \mathrm{CDCl}_{3}\right) \delta 171.9,160.8,148.3$, 143.7, 137.4, 129.2, 129.1, 127.6, 124.6, 124.5, 121.6, 119.9, 116.6, 114.2, 71.5, 55.4, 49.4, 18.4; HRMS (ESI) calcd for $\mathrm{C}_{24} \mathrm{H}_{24} \mathrm{~N}_{3} \mathrm{O}_{2} 386.1863\left[\mathrm{M}+\mathrm{H}^{+}\right]$, found 386.1858 .

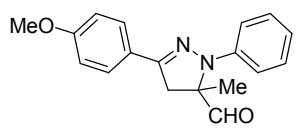

3-(4-methoxyphenyl)-5-methyl-1-phenyl-4,5-dihydro-1 $H$-pyrazole-5carbaldehyde (Table 2, entry 9): ${ }^{1} \mathrm{H}$ NMR $\left(400 \mathrm{MHz}, \mathrm{CDCl}_{3}\right) \delta 9.88(\mathrm{~s}, 1 \mathrm{H}), 7.67$ $(\mathrm{d}, J=8.8 \mathrm{~Hz}, 2 \mathrm{H}), 7.26(\mathrm{t}, J=8.8 \mathrm{~Hz}, 2 \mathrm{H}), 7.09(\mathrm{~d}, J=8.0 \mathrm{~Hz}, 2 \mathrm{H}), 6.95-6.90$ $(\mathrm{m}, 3 \mathrm{H}), 3.85(\mathrm{~s}, 3 \mathrm{H}), 3.48(\mathrm{~d}, J=16.8 \mathrm{~Hz}, 1 \mathrm{H}), 3.08(\mathrm{~d}, J=16.8 \mathrm{~Hz}, 1 \mathrm{H}), 1.44(\mathrm{~s}, 3 \mathrm{H}) ;{ }^{13} \mathrm{C} \mathrm{NMR}$ $\left(75.4 \mathrm{MHz}, \mathrm{CDCl}_{3}\right) \delta 199.9,160.5,146.6,144.0,129.2,127.4,124.7,120.7,115.2,114.2,74.3,55.4$, 43.8, 16.6; HRMS (ESI) calcd for $\mathrm{C}_{18} \mathrm{H}_{19} \mathrm{~N}_{2} \mathrm{O}_{2} 295.1441\left[\mathrm{M}+\mathrm{H}^{+}\right]$, found 295.1436.

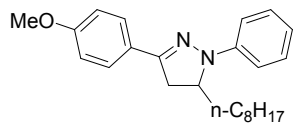

3-(4-methoxyphenyl)-5-octyl-1-phenyl-4,5-dihydro-1 $\boldsymbol{H}$-pyrazole (Table 2, entry 10): ${ }^{1} \mathrm{H}$ NMR $\left(500 \mathrm{MHz}, \mathrm{C}_{6} \mathrm{D}_{6}\right) \delta 7.70(\mathrm{~d}, J=9.0 \mathrm{~Hz}, 2 \mathrm{H}), 7.40(\mathrm{~d}, J=8.0$ $\mathrm{Hz}, 2 \mathrm{H}), 7.33(\mathrm{t}, J=8.5 \mathrm{~Hz}, 2 \mathrm{H}), 6.88-6.83(\mathrm{~m}, 3 \mathrm{H}), 4.00-3.92(\mathrm{~m}, 1 \mathrm{H}), 3.30(\mathrm{~s}$, $3 \mathrm{H}), 2.82((\mathrm{dd}, J=17.0,11.5,1 \mathrm{H}), 2.53(\mathrm{dd}, J=16.5,5.0 \mathrm{~Hz}, 1 \mathrm{H}), 1.65(\mathrm{br}, 1 \mathrm{H}), 1.36-1.06(\mathrm{~m}, 15 \mathrm{H})$, $0.89(\mathrm{t}, J=7.0 \mathrm{~Hz}, 1 \mathrm{H}) ;{ }^{13} \mathrm{C}$ NMR $\left(75.4 \mathrm{MHz}, \mathrm{C}_{6} \mathrm{D}_{6}\right) \delta 160.5,147.1,145.4,129.4,127.4,127.1$, $118.9,114.3,113.7,59.8,54.8,38.3,32.6,32.2,29.84,29.77,29.6,25.1,23.0,14.3$; HRMS (EI) calcd for $\mathrm{C}_{24} \mathrm{H}_{32} \mathrm{~N}_{2} \mathrm{O} 364.2509\left[\mathrm{M}^{+}\right]$, found 364.2510 .

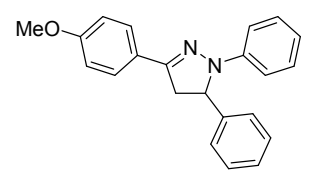

3-(4-methoxyphenyl)-1,5-diphenyl-4,5-dihydro-1 $\boldsymbol{H}$-pyrazole (Table 2, entry 11): ${ }^{1} \mathrm{H}$ NMR $\left(500 \mathrm{MHz}, \mathrm{CDCl}_{3}\right) \delta 7.66(\mathrm{~d}, J=8.5 \mathrm{~Hz}, 2 \mathrm{H}), 7.32(\mathrm{~d}, J=4.5 \mathrm{~Hz}$, 4H), 7.27-7.24 (m, 1H), 7.18-7.15 (m, 2H), $7.05(\mathrm{~d}, J=9.5 \mathrm{~Hz}, 2 \mathrm{H}), 6.92-6.89$ $(\mathrm{m}, 1 \mathrm{H}), 6.76(\mathrm{t}, J=7.5 \mathrm{~Hz}, 1 \mathrm{H}), 5.21(\mathrm{dd}, J=7.5,4.5 \mathrm{~Hz}, 1 \mathrm{H}), 3.83(\mathrm{~s}, 3 \mathrm{H})$, $3.80(\mathrm{dd}, J=17.0,4.5 \mathrm{~Hz}, 1 \mathrm{H}), 3.10(\mathrm{dd}, J=17.0,7.0 \mathrm{~Hz}, 1 \mathrm{H}) ;{ }^{13} \mathrm{C} \mathrm{NMR}\left(75.4 \mathrm{MHz}, \mathrm{CDCl}_{3}\right) \delta$ $160.1,146.7,145.2,142.8,129.1,128.8,127.5,127.2,125.9,125.5,118.8,114.0,113.3,64.5,55.3$, 43.8 .

$\mathrm{MeO}$

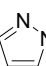

3-(4-methoxyphenyl)-1,5-diphenyl-1H-pyrazole (Table 2 , entry 11 , oxidation product): ${ }^{1} \mathrm{H}$ NMR $\left(500 \mathrm{MHz}, \mathrm{C}_{6} \mathrm{D}_{6}\right) \delta 8.10(\mathrm{~d}, J=9.0 \mathrm{~Hz}, 2 \mathrm{H}), 7.37(\mathrm{~d}, J=7.5$ $\mathrm{Hz}, 2 \mathrm{H}), 7.00-6.85(\mathrm{~m}, 9 \mathrm{H}), 6.69(\mathrm{~s}, 1 \mathrm{H}), 3.31(\mathrm{~s}, 3 \mathrm{H}) ;{ }^{13} \mathrm{C} \mathrm{NMR}(75.4 \mathrm{MHz}$, 
$\left.\mathrm{C}_{6} \mathrm{D}_{6}\right) \delta 128.8,128.5,127.7,127.5,127.4,127.2,126.7,126.5,125.1,114.2,105.1,54.5$.

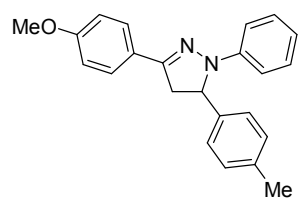

3-(4-methoxyphenyl)-1-phenyl-5-p-tolyl-4,5-dihydro-1 $\boldsymbol{H}$-pyrazole (Table 2, entry 12): ${ }^{1} \mathrm{H}$ NMR $\left(500 \mathrm{MHz}, \mathrm{CDCl}_{3}\right) \delta 7.65(\mathrm{~d}, J=8.0 \mathrm{~Hz}, 2 \mathrm{H}), 7.21(\mathrm{~d}, J=7.5$ $\mathrm{Hz}, 2 \mathrm{H}), 7.16(\mathrm{t}, J=7.5 \mathrm{~Hz}, 2 \mathrm{H}), 7.13(\mathrm{~d}, J=7.5 \mathrm{~Hz}, 2 \mathrm{H}), 7.05(\mathrm{~d}, J=8.0 \mathrm{~Hz}$, $2 \mathrm{H}), 6.90(\mathrm{~d}, J=8.0 \mathrm{~Hz}, 2 \mathrm{H}), 6.75(\mathrm{t}, J=7.0 \mathrm{~Hz}, 1 \mathrm{H}), 5.18(\mathrm{dd}, J=12.5,7.0 \mathrm{~Hz}$, $1 \mathrm{H}), 3.83(\mathrm{~s}, 3 \mathrm{H}), 3.78(\mathrm{dd}, J=17.0,12.5 \mathrm{~Hz}, 1 \mathrm{H}), 3.08(\mathrm{dd}, J=16.5,7.0 \mathrm{~Hz}, 1 \mathrm{H}), 2.31(\mathrm{~s}, 3 \mathrm{H}) ;{ }^{13} \mathrm{C}$ $\operatorname{NMR}\left(75.4 \mathrm{MHz}, \mathrm{CDCl}_{3}\right) \delta 160.1,146.7,145.2,139.8,137.1,129.7,128.8,127.2,125.8,125.6$, $118.7,114.0,113.2,64.3,55.3,43.9,21.1$.

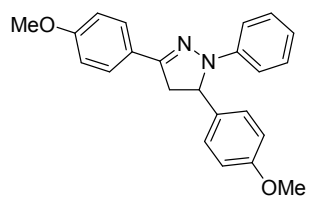

3,5-bis(4-methoxyphenyl)-1-phenyl-4,5-dihydro-1 $\boldsymbol{H}$-pyrazole (Table 2 , entry 13): ${ }^{1} \mathrm{H}$ NMR $\left(500 \mathrm{MHz}, \mathrm{CDCl}_{3}\right) \delta 7.66(\mathrm{~d}, J=9.0 \mathrm{~Hz}, 2 \mathrm{H}), 7.24(\mathrm{~d}, J=8.5 \mathrm{~Hz}$, $2 \mathrm{H}), 7.16(\mathrm{t}, J=7.5 \mathrm{~Hz}, 2 \mathrm{H}), 7.05(\mathrm{~d}, J=8.5 \mathrm{~Hz}, 2 \mathrm{H}), 6.91(\mathrm{~d}, J=8.5 \mathrm{~Hz}, 2 \mathrm{H})$, $6.85(\mathrm{~d}, J=8.5 \mathrm{~Hz}, 2 \mathrm{H}), 6.75(\mathrm{t}, J=7.0 \mathrm{~Hz}, 1 \mathrm{H}), 5.17(\mathrm{dd}, J=7.5,4.5 \mathrm{~Hz}, 1 \mathrm{H})$, $3.83(\mathrm{~s}, 3 \mathrm{H}), 3.77(\mathrm{~s}, 3 \mathrm{H}), 3.77(\mathrm{dd}, J=16.5,4.5 \mathrm{~Hz}, 1 \mathrm{H}), 3.08(\mathrm{dd}, J=16.5,7.0 \mathrm{~Hz}, 1 \mathrm{H}) ;{ }^{13} \mathrm{C} \mathrm{NMR}$ $\left(75.4 \mathrm{MHz}, \mathrm{CDCl}_{3}\right) \delta 160.1,158.9,146.8,145.2,134.8,128.8,127.2,127.1,125.6,118.8,114.4$, $114.0,113.3,64.0,55.3,55.2,43.9$.

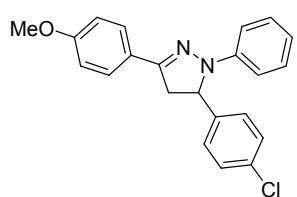

5-(4-chlorophenyl)-3-(4-methoxyphenyl)-1-phenyl-4,5-dihydro-1 $H$-pyrazole (Table 2, entry 14): ${ }^{1} \mathrm{H}$ NMR (500MHz, $\left.\mathrm{C}_{6} \mathrm{D}_{6}\right) \delta 7.62(\mathrm{~d}, J=8.5 \mathrm{~Hz}, 2 \mathrm{H}), 7.26(\mathrm{~d}$, $J=8.0 \mathrm{~Hz}, 2 \mathrm{H}), 7.20(\mathrm{t}, J=8.5 \mathrm{~Hz}, 2 \mathrm{H}), 6.97(\mathrm{~d}, J=8.5 \mathrm{~Hz}, 2 \mathrm{H}), 6.80$ (q, $J=8.5$ $\mathrm{Hz}, 5 \mathrm{H}), 4.61(\mathrm{dd}, J=12.0,7.0 \mathrm{~Hz}, 1 \mathrm{H}), 3.29(\mathrm{~s}, 3 \mathrm{H}), 3.04(\mathrm{dd}, J=17.0,12.5 \mathrm{~Hz}$, $1 \mathrm{H}), 2.51(\mathrm{dd}, J=16.5,7.5 \mathrm{~Hz}, 1 \mathrm{H}) ;{ }^{13} \mathrm{C} \mathrm{NMR}\left(75.4 \mathrm{MHz}, \mathrm{C}_{6} \mathrm{D}_{6}\right) \delta 160.7,146.7,145.6,141.6,133.3$, $129.4,129.3,127.61,127.59,126.0,119.5,114.4,113.8,64.0,54.8,43.6$.

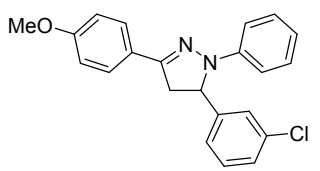

\section{5-(3-chlorophenyl)-3-(4-methoxyphenyl)-1-phenyl-4,5-dihydro-1 $H$-pyrazole} (Table 2, entry 15): ${ }^{1} \mathrm{H}$ NMR $\left(500 \mathrm{MHz}, \mathrm{C}_{6} \mathrm{D}_{6}\right) \delta 7.60(\mathrm{~d}, J=8.5 \mathrm{~Hz}, 2 \mathrm{H}), 7.26(\mathrm{~d}$, $J=8.0 \mathrm{~Hz}, 2 \mathrm{H}), 7.17(\mathrm{~d}, J=7.0 \mathrm{~Hz}, 2 \mathrm{H}), 6.93(\mathrm{dd}, J=8.0,1.0 \mathrm{~Hz}, 1 \mathrm{H}), 6.86(\mathrm{~d}$, $J=8.0 \mathrm{~Hz}, 1 \mathrm{H}), 6.85-6.77(\mathrm{~m}, 4 \mathrm{H}), 6.66(\mathrm{t}, J=8.0 \mathrm{~Hz}, 1 \mathrm{H}), 4.59(\mathrm{dd}, J=12.5$, $7.5 \mathrm{~Hz}, 1 \mathrm{H}), 4.59$ (dd, $J=12.5,7.5 \mathrm{~Hz}, 1 \mathrm{H}), 3.29$ (s, 3H), 3.00 (dd, $J=16.5,12.5 \mathrm{~Hz}, 1 \mathrm{H}), 2.48$ (dd, $J=17.0,7.5 \mathrm{~Hz}, 1 \mathrm{H}) ;{ }^{13} \mathrm{C} \mathrm{NMR}\left(75.4 \mathrm{MHz}, \mathrm{C}_{6} \mathrm{D}_{6}\right) \delta 160.8,146.9,145.7,145.4,135.1,130.7,129.3$, 127.7, 126.4, 125.9, 124.1, 119.6, 114.3, 113.8, 64.1, 54.8, 43.5; HRMS (EI) calcd for $\mathrm{C}_{22} \mathrm{H}_{19} \mathrm{~N}_{2} \mathrm{OCl}$ $362.1180\left[\mathrm{M}^{+}\right]$, found 362.1164 .

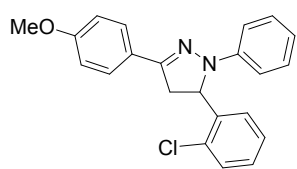

5-(2-chlorophenyl)-3-(4-methoxyphenyl)-1-phenyl-4,5-dihydro-1 $H$-pyrazole (Table 2, entry 16): ${ }^{1} \mathrm{H}$ NMR $\left(500 \mathrm{MHz}, \mathrm{C}_{6} \mathrm{D}_{6}\right) \delta 7.57(\mathrm{~d}, J=9.0 \mathrm{~Hz}, 2 \mathrm{H}), 7.28(\mathrm{~d}$, $J=8.0 \mathrm{~Hz}, 2 \mathrm{H}), 7.20-7.16(\mathrm{~m}, 4 \mathrm{H}), 6.79-6.76(\mathrm{~m}, 3 \mathrm{H}), 6.90-6.62(\mathrm{~m}, 2 \mathrm{H}), 5.37$ $(\mathrm{dd}, J=12.5,7.0 \mathrm{~Hz}, 1 \mathrm{H}), 3.32(\mathrm{dd}, J=16.5,7.0 \mathrm{~Hz}, 1 \mathrm{H}), 3.28(\mathrm{~s}, 3 \mathrm{H}), 2.59$ (dd, $J=17.0,7.0 \mathrm{~Hz}, 1 \mathrm{H}) ;{ }^{13} \mathrm{C} \mathrm{NMR}\left(75.4 \mathrm{MHz}, \mathrm{C}_{6} \mathrm{D}_{6}\right) \delta 160.7,147.1,145.4,140.1,132.0,129.9,129.4$, $128.8,127.7,126.0,119.4,114.3,113.6,61.5,54.8,42.2$.

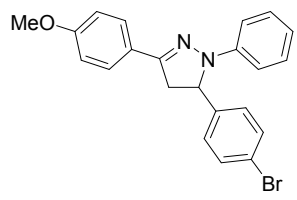

5-(4-bromophenyl)-3-(4-methoxyphenyl)-1-phenyl-4,5-dihydro-1 $H$-pyrazole (Table 2, entry 17): ${ }^{1} \mathrm{H}$ NMR $\left(500 \mathrm{MHz}, \mathrm{C}_{6} \mathrm{D}_{6}\right) \delta 7.62(\mathrm{~d}, J=9.0 \mathrm{~Hz}, 2 \mathrm{H}), 7.26(\mathrm{~d}$, $J=7.5 \mathrm{~Hz}, 2 \mathrm{H}), 7.21-7.10(\mathrm{~m}, 4 \mathrm{H}), 6.82(\mathrm{~d}, J=10.0 \mathrm{~Hz}, 2 \mathrm{H}), 6.72(\mathrm{~d}, J=9.0 \mathrm{~Hz}$, 
2H), $4.58(\mathrm{dd}, J=12.5,7.5 \mathrm{~Hz}, 1 \mathrm{H}), 3.29(\mathrm{~s}, 3 \mathrm{H}), 3.03(\mathrm{dd}, J=16.5,12.5 \mathrm{~Hz}, 1 \mathrm{H}), 2.50(\mathrm{dd}, J=17.0$, $7.5 \mathrm{~Hz}, 1 \mathrm{H}) ;{ }^{13} \mathrm{C} \mathrm{NMR}\left(75.4 \mathrm{MHz}, \mathrm{C}_{6} \mathrm{D}_{6}\right) \delta 160.8,146.7,145.6,142.1,132.4,129.3,127.7,125.9$, $121.4,119.6,114.4,113.8,64.0,54.8,43.5$.

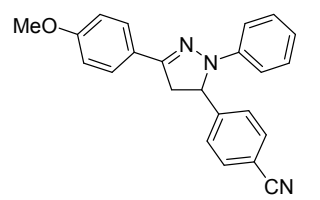

\section{4-(3-(4-methoxyphenyl)-1-phenyl-4,5-dihydro-1H-pyrazol-5-yl)benzonitrile} (Table 2, entry 18): ${ }^{1} \mathrm{H}$ NMR $\left(500 \mathrm{MHz}, \mathrm{C}_{6} \mathrm{D}_{6}\right) \delta 7.60(\mathrm{~d}, J=9.0 \mathrm{~Hz}, 2 \mathrm{H})$, $7.21-7.15(\mathrm{~m}, 4 \mathrm{H}), 6.88(\mathrm{~d}, J=8.5 \mathrm{~Hz}, 2 \mathrm{H}), 6.83-6.81(\mathrm{~m}, 3 \mathrm{H}), 6.68(\mathrm{~d}, J=8.0$ $\mathrm{Hz}, 2 \mathrm{H}), 4.51(\mathrm{dd}, J=12.5,7.5 \mathrm{~Hz}, 1 \mathrm{H}), 3.28(\mathrm{~s}, 3 \mathrm{H}), 2.98(\mathrm{dd}, J=17.0,12.5 \mathrm{~Hz}$, $1 \mathrm{H}), 2.38(\mathrm{dd}, J=17.0,7.5 \mathrm{~Hz}, 1 \mathrm{H}) ;{ }^{13} \mathrm{C} \mathrm{NMR}\left(75.4 \mathrm{MHz}, \mathrm{C}_{6} \mathrm{D}_{6}\right) \delta 163.1,160.9,147.6,146.7,145.4$, 132.8, 129.3, 126.6, 125.6, 119.8, 118.5, 114.4, 113.7, 112.0, 64.1, 54.9, 43.3; HRMS (EI) calcd for $\mathrm{C}_{23} \mathrm{H}_{20} \mathrm{~N}_{3} \mathrm{O} 353.1523\left[\mathrm{M}^{+}\right]$, found 353.1526.

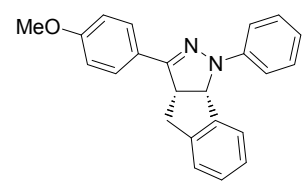

$(3 \mathrm{aS} *, 8 \mathrm{bS} *)-3-(4-m e t h o x y p h e n y l)-1-p h e n y l-1,3 a, 4,8 b-t e t r a h y d r o i n d e n o[1,2-$ clpyrazole (Table 2, entry 19 , racemic form): ${ }^{1} \mathrm{H}$ NMR $\left(500 \mathrm{MHz}, \mathrm{C}_{6} \mathrm{D}_{6}\right) \delta 7.57$ (dd, $J=15.0,8.5 \mathrm{~Hz}, 4 \mathrm{H}), 7.34(\mathrm{t}, J=8.0 \mathrm{~Hz}, 2 \mathrm{H}), 6.97(\mathrm{t}, J=7.5 \mathrm{~Hz}, 2 \mathrm{H}), 6.90$ $(\mathrm{t}, J=8.0 \mathrm{~Hz}, 2 \mathrm{H}), 6.85(\mathrm{~d}, J=7.0 \mathrm{~Hz}, 1 \mathrm{H}), 6.81(\mathrm{~d}, J=9.0 \mathrm{~Hz}, 2 \mathrm{H}), 5.47(\mathrm{~d}, J=$ $10.5 \mathrm{~Hz}, 1 \mathrm{H}), 3.76(\mathrm{td}, J=7.0,1.0 \mathrm{~Hz}, 1 \mathrm{H}), 3,29(\mathrm{~s}, 3 \mathrm{H}), 3.02-2.88(\mathrm{~m}, 2 \mathrm{H}) ;{ }^{13} \mathrm{C} \mathrm{NMR}(75.4 \mathrm{MHz}$, $\left.\mathrm{C}_{6} \mathrm{D}_{6}\right) \delta 160.4,150.5,145.6,142.1,141.6,129.5,128.6,127.5,126.0,125.4,124.9,119.2,114.3$, 113.9, 69.2, 54.8, 48.9, 36.3; HRMS (ESI) calcd for $\mathrm{C}_{23} \mathrm{H}_{21} \mathrm{~N}_{2} \mathrm{O} 341.1648\left[\mathrm{M}+\mathrm{H}^{+}\right]$, found 341.1636.

\section{Characterization of tetrazoles and pyrazolines described in Table 3}

The yields of tetrazole formation were based on the corresponding aldehyde starting materials.

5-(4-methoxyphenyl)-2-p-tolyl-2H-tetrazole (Table 3, entry 2): 52\% yield; 1b ${ }^{1} \mathrm{H}$ NMR $\left(400 \mathrm{MHz}, \mathrm{CDCl}_{3}\right) \delta 8.21(\mathrm{~d}, J=8.4 \mathrm{~Hz}, 2 \mathrm{H}), 8.08(\mathrm{~d}, J=8.4 \mathrm{~Hz}$, 2H), $7.38(\mathrm{~d}, J=8.0 \mathrm{~Hz}, 2 \mathrm{H}), 7.06(\mathrm{~d}, J=8.4 \mathrm{~Hz}, 2 \mathrm{H}), 3.91(\mathrm{~s}, 3 \mathrm{H}), 2.48(\mathrm{~s}, 3 \mathrm{H}) ;{ }^{13} \mathrm{C}$ NMR $(75.4$ $\left.\mathrm{MHz}, \mathrm{CDCl}_{3}\right) \delta 165.4,161.9,140.2,135.3,130.6,129.1,120.4,120.2,114.8,55.9,21.7$.

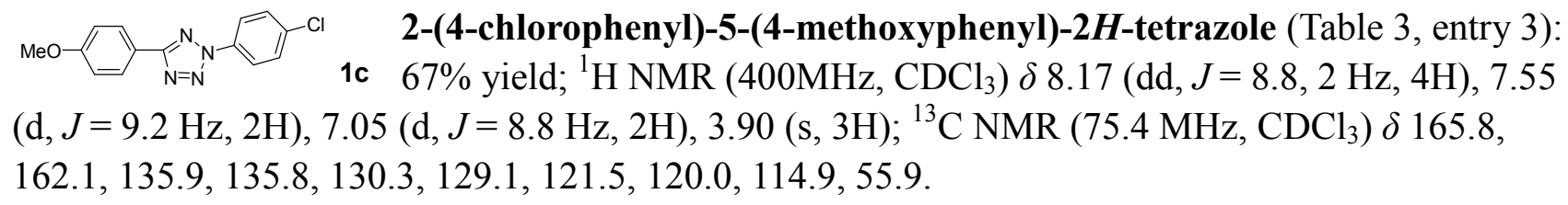

2-(4-bromophenyl)-5-(4-methoxyphenyl)-2H-tetrazole (Table 3, entry 4): 1d $45 \%$ yield; ${ }^{1} \mathrm{H} \mathrm{NMR}\left(400 \mathrm{MHz}, \mathrm{CDCl}_{3}\right) \delta 8.18(\mathrm{~d}, J=8.8 \mathrm{~Hz}, 2 \mathrm{H}), 8.09(\mathrm{~d}, J$ $=8.8 \mathrm{~Hz}, 2 \mathrm{H}), 7.71(\mathrm{~d}, J=8.8 \mathrm{~Hz}, 2 \mathrm{H}), 7.04(\mathrm{~d}, J=8.8 \mathrm{~Hz}, 2 \mathrm{H}), 3.89(\mathrm{~s}, 3 \mathrm{H}) ;{ }^{13} \mathrm{C} \mathrm{NMR}(75.4 \mathrm{MHz}$, $\left.\mathrm{CDCl}_{3}\right) \delta 165.8,162.1,136.4,133.3,129.1,123.8,121.7,120.0,114.9,55.9$.

\footnotetext{
2-phenyl-5-p-tolyl-2H-tetrazole (Table 3, entry 5): 61\% yield; ${ }^{1} \mathrm{H}$ NMR 1e $\left(300 \mathrm{MHz}, \mathrm{CDCl}_{3}\right) \delta 8.20(\mathrm{~d}, J=7.8 \mathrm{~Hz}, 2 \mathrm{H}), 8.14(\mathrm{~d}, J=8.1 \mathrm{~Hz}, 2 \mathrm{H}), 7.58(\mathrm{t}$, $J=7.2 \mathrm{~Hz}, 2 \mathrm{H}), 7.50$ (t, $J=7.2 \mathrm{~Hz}, 1 \mathrm{H}), 7.34(\mathrm{~d}, J=7.8 \mathrm{~Hz}), 2.44(\mathrm{~s}, 3 \mathrm{H}) ;{ }^{13} \mathrm{C}$ NMR $(75.4 \mathrm{MHz}$, $\left.\mathrm{CDCl}_{3}\right) \delta 165.8,141.3,137.5,130.2,130.1,130.0,127.5,124.9,120.4,22.0$.
} 


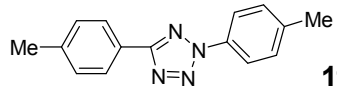

2,5-di-p-tolyl-2H-tetrazole (Table 3, entry 6): $52 \%$ yield; ${ }^{1} \mathrm{H}$ NMR $(300 \mathrm{MHz}$, If $\left.\mathrm{CDCl}_{3}\right) \delta 8.13(\mathrm{~d}, J=8.1 \mathrm{~Hz}, 2 \mathrm{H}), 8.06(\mathrm{~d}, J=8.4 \mathrm{~Hz}, 2 \mathrm{H}), 7.34(\mathrm{t}, J=8.7 \mathrm{~Hz}$, $4 \mathrm{H}), 2.45(\mathrm{~s}, 3 \mathrm{H}), 2.43(\mathrm{~s}, 3 \mathrm{H}) ;{ }^{13} \mathrm{C} \mathrm{NMR}\left(75.4 \mathrm{MHz} \mathrm{CDCl}_{3}\right) \delta 165.7,141.2,140.3,135.3,130.6$, $130.1,127.5,125.0,120.3,22.0,21.7$.

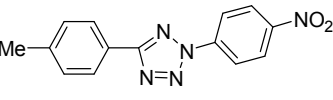

2-(4-nitrophenyl)-5-p-tolyl-2H-tetrazole (Table 3, entry 7): $22 \%$ yield; ${ }^{1} \mathrm{H}$ $1 \mathrm{~N} \quad \mathrm{NMR}\left(300 \mathrm{MHz}, \mathrm{CDCl}_{3}\right) \delta 8.63(\mathrm{dd}, J=9.3,6.0 \mathrm{~Hz}, 4 \mathrm{H}), 8.34(\mathrm{~d}, J=8.4$ $\mathrm{Hz}, 2 \mathrm{H}), 7.55(\mathrm{~d}, J=8.1 \mathrm{~Hz}, 2 \mathrm{H}), 2.65(\mathrm{~s}, 3 \mathrm{H}) ;{ }^{13} \mathrm{C} \mathrm{NMR}\left(75.4 \mathrm{MHz}, \mathrm{CDCl}_{3}\right): \delta 166.6,148.3,142.0$, $141.2,130.3,127.7,125.9,124.1,120.7,22.1$.

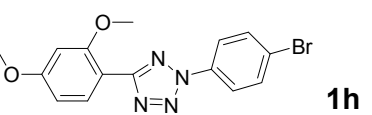

2-(4-bromophenyl)-5-(2,4-dimethoxyphenyl)-2H-tetrazole (Table 3, entry 8): $59 \%$ yield; ${ }^{1} \mathrm{H}$ NMR $\left(400 \mathrm{MHz}, \mathrm{CDCl}_{3}\right) \delta 8.09(\mathrm{~d}, J=8.8 \mathrm{~Hz}, 2 \mathrm{H}), 8.01$ $(\mathrm{d}, J=8.4 \mathrm{~Hz}, 1 \mathrm{H}), 7.70(\mathrm{~d}, J=8.8 \mathrm{~Hz}, 2 \mathrm{H}), 6.65(\mathrm{dd}, J=10.8,2.0 \mathrm{~Hz}, 1 \mathrm{H})$, $6.62(\mathrm{~d}, J=2.4 \mathrm{~Hz}, 1 \mathrm{H}), 3.96(\mathrm{~s}, 3 \mathrm{H}), 3.90(\mathrm{~s}, 3 \mathrm{H}) ;{ }^{13} \mathrm{C} \mathrm{NMR}\left(75.4 \mathrm{MHz}, \mathrm{CDCl}_{3}\right) \delta 164.2,163.4$, 159.6, 136.5, 133.2, 132.3, 123.7, 121.9, 109.5, 105.8, 99.8, 56.5, 56.0; HRMS (ESI) calcd for $\mathrm{C}_{15} \mathrm{H}_{13} \mathrm{~N}_{4} \mathrm{O}_{2} \mathrm{BrNa}\left({ }^{79} \mathrm{Br}\right) 383.0114\left[\mathrm{M}+\mathrm{Na}^{+}\right]$, found 383.0122 .

2-(4-chlorophenyl)-5-(2,4-dimethoxyphenyl)-2H-tetrazole (Table 3, entry 9): $67 \%$ yield; ${ }^{1} \mathrm{H}$ NMR $\left(300 \mathrm{MHz}, \mathrm{CDCl}_{3}\right) \delta 8.15(\mathrm{~d}, J=8.7 \mathrm{~Hz}, 2 \mathrm{H}), 8.00$ 1i $(\mathrm{d}, J=8.4 \mathrm{~Hz}, 1 \mathrm{H}), 7.53(\mathrm{~d}, J=8.7 \mathrm{~Hz}, 1 \mathrm{H}), 6.66(\mathrm{~d}, J=2.4 \mathrm{~Hz}, 1 \mathrm{H}), 6.62(\mathrm{~s}$, $1 \mathrm{H}) ;{ }^{13} \mathrm{C}$ NMR $\left(75.4 \mathrm{MHz}, \mathrm{CDCl}_{3}\right) \delta 163.6,162.8,159.0,135.5,135.2,131.8,129.7,121.1,109.0$, 105.2, 99.2, 56.0, 55.5; HRMS (ESI) calcd for $\mathrm{C}_{15} \mathrm{H}_{14} \mathrm{~N}_{4} \mathrm{O}_{2} \mathrm{Cl}\left({ }^{35} \mathrm{Cl}\right) 317.0800\left[\mathrm{M}+\mathrm{H}^{+}\right]$, found 317.0795 .

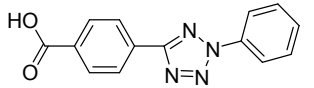

4-(2-phenyl-2H-tetrazol-5-yl)benzoic acid (Table 3, entry 10$): 34 \%$ yield; ${ }^{1} \mathrm{H}$ 1j NMR (500MHz, DMSO-d $\left.{ }_{6}\right) \delta 12.29(\mathrm{~s}, 1 \mathrm{H}), 8.32(\mathrm{~d}, J=8.0 \mathrm{~Hz}, 2 \mathrm{H}), 8.19(\mathrm{t}$, $J=8.5 \mathrm{~Hz}, 4 \mathrm{H}), 7.73(\mathrm{t}, J=7.5 \mathrm{~Hz}, 2 \mathrm{H}), 7.66(\mathrm{t}, J=7.5 \mathrm{~Hz}, 1 \mathrm{H}) ;{ }^{13} \mathrm{C}$ NMR $\left(75.4 \mathrm{MHz}, \mathrm{DMSO}-\mathrm{d}_{6}\right) \delta$ 166.7, 163.8, 136.1, 132.8, 130.4, 130.3, 130.2, 126.8, 120.0; HRMS (ESI) calcd for $\mathrm{C}_{14} \mathrm{H}_{11} \mathrm{~N}_{4} \mathrm{O}_{2}$ $267.0877\left[\mathrm{M}+\mathrm{H}^{+}\right]$, found 267.0875 .

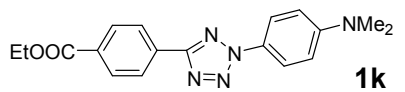

Ethyl 4-(2-(4-(dimethylamino)phenyl)-2H-tetrazol-5-yl)benzoate (Table 3, entry 11$): 13 \%$ yield; ${ }^{1} \mathrm{H}$ NMR $\left(400 \mathrm{MHz}, \mathrm{CDCl}_{3}\right) \delta 8.17(\mathrm{dd}, J=5.2,1.6$ $\mathrm{Hz}, 2 \mathrm{H}), 7.58(\mathrm{dd}, J=4.8,2.0 \mathrm{~Hz}, 2 \mathrm{H}), 4.58(\mathrm{q}, J=7.2 \mathrm{~Hz}, 2 \mathrm{H}), 1.50(\mathrm{t}, J=7.2 \mathrm{~Hz}, 3 \mathrm{H}) ;{ }^{13} \mathrm{C} \mathrm{NMR}$ $\left(75.4 \mathrm{MHz}, \mathrm{CDCl}_{3}\right) \delta 157.9,157.6,136.7,134.8,130.1,121.5,62.9,14.2$; HRMS (ESI) calcd for $\mathrm{C}_{10} \mathrm{H}_{10} \mathrm{~N}_{4} \mathrm{O}_{2} \mathrm{Cl}\left({ }^{35} \mathrm{Cl}\right) 253.0487\left[\mathrm{M}+\mathrm{H}^{+}\right]$, found 253.0479 .

2-(2-phenyl-2H-tetrazol-5-yl)pyridine (Table 3, entry 12): 53\% yield; ${ }^{1} \mathrm{H}$ NMR 1 I $\left(300 \mathrm{MHz}, \mathrm{CDCl}_{3}\right) \delta 7.77(\mathrm{~d}, J=7.5 \mathrm{~Hz}, 1 \mathrm{H}), 7.63(\mathrm{~d}, J=8.1 \mathrm{~Hz}, 1 \mathrm{H}), 7.52-7.31$ $(\mathrm{m}, 4 \mathrm{H}), 7.15(\mathrm{~d}, J=6.9 \mathrm{~Hz}, 2 \mathrm{H}), 6.91(\mathrm{~d}, J=6.3 \mathrm{~Hz}, 1 \mathrm{H}) ;{ }^{13} \mathrm{C} \mathrm{NMR}\left(75.4 \mathrm{MHz}, \mathrm{CDCl}_{3}\right) \delta 140.2$, $137.1,134.8,134.1,133.6,129.1,129.0,128.9,128.8,128.5,128.4,125.8$.

5-(4-nitrophenyl)-2-p-tolyl-2H-tetrazole (Table 3, entry 13): 59\% yield; $1 \mathrm{~m} \quad{ }^{1} \mathrm{H}$ NMR $\left(300 \mathrm{MHz}, \mathrm{CDCl}_{3}\right) \delta 8.39(\mathrm{dd}, J=9,9 \mathrm{~Hz}, 4 \mathrm{H}), 8.06(\mathrm{~d}, J=8.4$, $2 \mathrm{H}), 8.38(\mathrm{~d}, J=8.1 \mathrm{~Hz}, 2 \mathrm{H}), 2.46(\mathrm{~s}, 3 \mathrm{H}) ;{ }^{13} \mathrm{C} \mathrm{NMR}\left(75.4 \mathrm{MHz}, \mathrm{CDCl}_{3}\right): \delta 163.7,149.5,141.0$, 
$135.0,133.6,130.8,128.3,124.8,120.3,21.8$.

Ethyl 2-(4-chlorophenyl)-2H-tetrazole-5-carboxylate (Table 3, entry 14): 1n yield $37 \% ;{ }^{1} \mathrm{H}$ NMR $\left(400 \mathrm{MHz}, \mathrm{CDCl}_{3}\right) \delta 8.17(\mathrm{dd}, J=5.2,1.6 \mathrm{~Hz}, 2 \mathrm{H}), 7.58$ $(\mathrm{dd}, J=4.8,2.0 \mathrm{~Hz}, 2 \mathrm{H}), 4.58(\mathrm{q}, J=7.2 \mathrm{~Hz}, 2 \mathrm{H}), 1.50(\mathrm{t}, J=7.2 \mathrm{~Hz}, 3 \mathrm{H}),{ }^{13} \mathrm{C} \mathrm{NMR}(75.4 \mathrm{MHz}$, $\left.\mathrm{CDCl}_{3}\right) \delta 157.9,157.6,136.7,134.8,130.1,121.5,62.9,14.2$; HRMS (ESI) calcd for $\mathrm{C}_{10} \mathrm{H}_{10} \mathrm{~N}_{4} \mathrm{O}_{2} \mathrm{Cl}\left({ }^{35} \mathrm{Cl}\right) 253.0487\left[\mathrm{M}+\mathrm{H}^{+}\right]$, found 253.0479 .

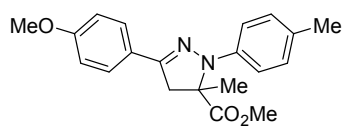

Methyl 3-(4-methoxyphenyl)-5-methyl-1-p-tolyl-4,5-dihydro-1 $H$-pyrazole5-carboxylate (Table 3 , entry 2$):{ }^{1} \mathrm{H} \mathrm{NMR}\left(400 \mathrm{MHz}, \mathrm{CDCl}_{3}\right) \delta 7.63(\mathrm{~d}, J=$ $8.4 \mathrm{~Hz}, 2 \mathrm{H}), 7.06(\mathrm{~d}, J=8.4 \mathrm{~Hz}, 2 \mathrm{H}), 6.99(\mathrm{~d}, J=8.8 \mathrm{~Hz}, 2 \mathrm{H}), 6.91(\mathrm{~d}, J=8.4$ $\mathrm{Hz}, 2 \mathrm{H}), 3.84(\mathrm{~s}, 3 \mathrm{H}), 3.76(\mathrm{~s}, 3 \mathrm{H}), 3.65(\mathrm{~d}, J=16.4 \mathrm{~Hz}, 1 \mathrm{H}), 3.24(\mathrm{~d}, J=16.4 \mathrm{~Hz}, 1 \mathrm{H}), 2.23(\mathrm{~s}, 3 \mathrm{H})$, 1.59 (s, 3H); ${ }^{13} \mathrm{C}$ NMR $\left(75.4 \mathrm{MHz}, \mathrm{CDCl}_{3}\right) \delta 174.5,160.1,144.8,141.4,129.5,129.4,127.1,125.3$, $115.0,114.0,69.2,55.3,52.9,48.2,20.9,20.5$.

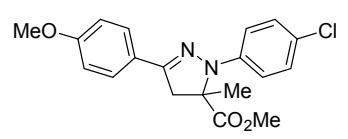

Methyl 1-(4-chlorophenyl)-3-(4-methoxyphenyl)-5-methyl-4,5-dihydro-1 Hpyrazole-5-carboxylate (Table 3 , entry 3 ): ${ }^{1} \mathrm{H}$ NMR $\left(400 \mathrm{MHz}, \mathrm{CDCl}_{3}\right) \delta 7.63$ $(\mathrm{d}, J=8.4 \mathrm{~Hz}, 2 \mathrm{H}), 7.19(\mathrm{~d}, J=8.8 \mathrm{~Hz}, 2 \mathrm{H}), 7.02(\mathrm{~d}, J=8.4 \mathrm{~Hz}, 2 \mathrm{H}), 6.92(\mathrm{~d}$, $J=8.4 \mathrm{~Hz}, 2 \mathrm{H}), 3.85(\mathrm{~s}, 3 \mathrm{H}), 3.76(\mathrm{~s}, 3 \mathrm{H}), 3.68(\mathrm{~d}, J=16.8 \mathrm{~Hz}, 1 \mathrm{H}), 3.27(\mathrm{~d}, J=17.2 \mathrm{~Hz}, 1 \mathrm{H}), 1.62$ $(\mathrm{s}, 3 \mathrm{H}) ;{ }^{13} \mathrm{C}$ NMR $\left(75.4 \mathrm{MHz}, \mathrm{CDCl}_{3}\right) \delta 174.1,160.4,145.8,142.2,128.9,127.3,124.8,124.6,115.6$, 114.1, 68.9, 55.4, 53.0, 48.4, 21.0; HRMS (ESI) calcd for $\mathrm{C}_{19} \mathrm{H}_{20} \mathrm{~N}_{2} \mathrm{O}_{3} \mathrm{Cl}\left({ }^{35} \mathrm{Cl}\right) 359.1157\left[\mathrm{M}+\mathrm{H}^{+}\right]$, found 359.1157 .

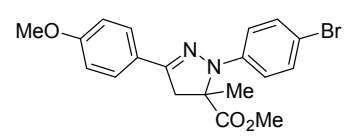

Methyl 1-(4-bromophenyl)-3-(4-methoxyphenyl)-5-methyl-4,5-dihydro-1 $H$ -pyrazole-5-carboxylate (Table 3 , entry 4): ${ }^{1} \mathrm{H}$ NMR $\left(300 \mathrm{MHz}, \mathrm{CDCl}_{3}\right) \delta$ $7.63(\mathrm{~d}, J=8.7 \mathrm{~Hz}, 2 \mathrm{H}), 7.33(\mathrm{~d}, J=8.7 \mathrm{~Hz}, 2 \mathrm{H}), 6.94(\mathrm{dd}, J=12.9,9.3 \mathrm{~Hz}$, $4 \mathrm{H}), 3.85(\mathrm{~s}, 3 \mathrm{H}), 3.76(\mathrm{~s}, 3 \mathrm{H}), 3.68(\mathrm{~d}, J=17.1 \mathrm{~Hz}, 1 \mathrm{H}), 3.27(\mathrm{~d}, J=16.8 \mathrm{~Hz}, 1 \mathrm{H}), 1.63(\mathrm{~s}, 3 \mathrm{H}) ;{ }^{13} \mathrm{C}$ NMR (75.4 MHz, $\left.\mathrm{CDCl}_{3}\right) \delta 174.0,160.5,145.8,142.6,131.8,127.3,124.7,115.9,114.1,111.9,68.7$, 55.4, 53.0, 48.5, 21.0; HRMS (ESI) calcd for $\mathrm{C}_{19} \mathrm{H}_{20} \mathrm{~N}_{2} \mathrm{O}_{3} \mathrm{Brl}\left({ }^{79} \mathrm{Br}\right) 403.0652\left[\mathrm{M}+\mathrm{H}^{+}\right]$, found 403.0654 .

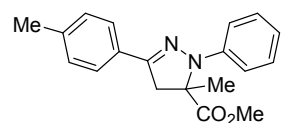

\section{Methyl 5-methyl-1-phenyl-3-p-tolyl-4,5-dihydro-1 $H$-pyrazole-5-carboxylate} (Table 3, entry 5): ${ }^{1} \mathrm{H}$ NMR $\left(400 \mathrm{MHz}, \mathrm{CDCl}_{3}\right) \delta 7.60(\mathrm{~d}, J=8.0 \mathrm{~Hz}, 2 \mathrm{H}), 7.25(\mathrm{t}$, $J=8.8 \mathrm{~Hz}, 2 \mathrm{H}), 7.20(\mathrm{~d}, J=7.6 \mathrm{~Hz}, 2 \mathrm{H}), 7.10(\mathrm{~d}, J=7.6 \mathrm{~Hz}, 2 \mathrm{H}), 6.87(\mathrm{t}, J=6.8$ $\mathrm{Hz}, 1 \mathrm{H}), 3.76(\mathrm{~s}, 3 \mathrm{H}), 3.68(\mathrm{~d}, J=16.8 \mathrm{~Hz}, 1 \mathrm{H}), 3.27$ (d, $J=16.8 \mathrm{~Hz}, 1 \mathrm{H}), 2.38(\mathrm{~s}, 3 \mathrm{H}), 1.64(\mathrm{~s}, 3 \mathrm{H})$; ${ }^{13} \mathrm{C} \mathrm{NMR}\left(75.4 \mathrm{MHz}, \mathrm{CDCl}_{3}\right) \delta 174.4,145.3,143.3,138.9,129.6,129.3,129.0,125.7,120.0,114.5$, $68.9,52.9,48.2,21.4,21.1$.

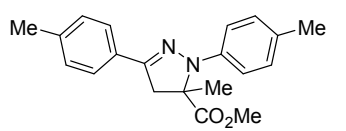

\section{Methyl 5-methyl-1,3-dip-tolyl-4,5-dihydro- $1 H$-pyrazole-5-carboxylate} (Table 3, entry 6): ${ }^{1} \mathrm{H}$ NMR $\left(400 \mathrm{MHz}, \mathrm{CDCl}_{3}\right) \delta 7.58(\mathrm{~d}, J=8.0 \mathrm{~Hz}, 2 \mathrm{H}), 7.19$ $(\mathrm{d}, J=8.0 \mathrm{~Hz}, 2 \mathrm{H}), 7.06(\mathrm{~d}, J=8.4 \mathrm{~Hz}, 2 \mathrm{H}), 7.00(\mathrm{~d}, J=8.4 \mathrm{~Hz}, 2 \mathrm{H}), 3.76(\mathrm{~s}$, $3 \mathrm{H}), 3.66(\mathrm{~d}, J=16.8 \mathrm{~Hz}, 1 \mathrm{H}), 3.25(\mathrm{~d}, J=16.4 \mathrm{~Hz}, 1 \mathrm{H}), 2.38(\mathrm{~s}, 3 \mathrm{H}), 2.28(\mathrm{~s}, 3 \mathrm{H}), 1.59(\mathrm{~s}, 3 \mathrm{H}) ;{ }^{13} \mathrm{C}$ NMR (75.4 MHz, $\left.\mathrm{CDCl}_{3}\right) \delta 174.5,145.0,141.3,138.7,129.7,129.5,129.2,125.6,115.1,69.2,52.9$, 48.1, 21.4, 21.0, 20.5; HRMS (ESI) calcd for $\mathrm{C}_{20} \mathrm{H}_{23} \mathrm{~N}_{2} \mathrm{O}_{2} 323.1754\left[\mathrm{M}+\mathrm{H}^{+}\right]$, found 323.1750. 


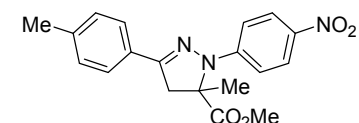

Methyl 5-methyl-1-(4-nitrophenyl)-3-p-tolyl-4,5-dihydro-1 $H$-pyrazole-5carboxylate (Table 3 , entry 7$):{ }^{1} \mathrm{H}$ NMR $\left(500 \mathrm{MHz}, \mathrm{CDCl}_{3}\right) \delta 8.14(\mathrm{~d}, J=9.0$ $\mathrm{Hz}, 2 \mathrm{H}), 7.62(\mathrm{~d}, J=8.0 \mathrm{~Hz}, 2 \mathrm{H}), 7.24(\mathrm{~d}, J=8.0 \mathrm{~Hz}, 2 \mathrm{H}), 7.08(\mathrm{~d}, J=9.0 \mathrm{~Hz}$, 2H), $3.78(\mathrm{~s}, 3 \mathrm{H}), 3.77(\mathrm{~d}, J=17.0 \mathrm{~Hz}, 1 \mathrm{H}), 3.37(\mathrm{~d}, J=17.0 \mathrm{~Hz}, 1 \mathrm{H}), 2.41(\mathrm{~s}, 3 \mathrm{H}), 1.76(\mathrm{~s}, 3 \mathrm{H}) ;{ }^{13} \mathrm{C}$ NMR $\left(75.4 \mathrm{MHz}, \mathrm{CDCl}_{3}\right) \delta 173.0,149.1,147.6,140.4,139.5,129.5,128.3,126.2,125.8,112.1,68.2$, 53.4, 48.8, 21.5, 21.4; HRMS (EI) calcd for $\mathrm{C}_{19} \mathrm{H}_{19} \mathrm{~N}_{3} \mathrm{O}_{4} 353.1370\left[\mathrm{M}^{+}\right]$, found 353.1372.

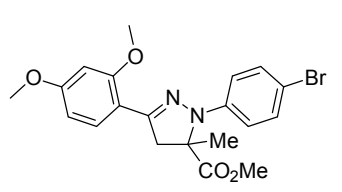

Methyl 1-(4-bromophenyl)-3-(2,4-dimethoxyphenyl)-5-methyl-4,5-dihydro$1 \boldsymbol{H}$-pyrazole-5-carboxylate (Table 3 , entry 8): ${ }^{1} \mathrm{H} \mathrm{NMR}\left(400 \mathrm{MHz}, \mathrm{CDCl}_{3}\right) \delta$ $7.90(\mathrm{~d}, J=8.8 \mathrm{~Hz}, 2 \mathrm{H}), 7.31(\mathrm{~d}, J=8.8 \mathrm{~Hz}, 2 \mathrm{H}), 6.94(\mathrm{~d}, J=8.8 \mathrm{~Hz}, 2 \mathrm{H}), 6.55$ $(\mathrm{dd}, J=8.8,2 \mathrm{~Hz}, 1 \mathrm{H}), 6.46(\mathrm{~d}, J=2.4 \mathrm{~Hz}, 1 \mathrm{H}), 7.90(\mathrm{~d}, J=8.8 \mathrm{~Hz}, 2 \mathrm{H}), 3.84$ (s, 3H), $3.83(\mathrm{~s}, 3 \mathrm{H}), 3.82(\mathrm{~d}, J=17.6 \mathrm{~Hz}, 1 \mathrm{H}), 3.76(\mathrm{~s}, 3 \mathrm{H}), 3.45(\mathrm{~d}, J=17.6 \mathrm{~Hz}, 1 \mathrm{H}), 1.59$ (s, 3H); ${ }^{13} \mathrm{C} \mathrm{NMR}\left(75.4 \mathrm{MHz}, \mathrm{CDCl}_{3}\right) \delta 174.4,161.8,158.7,145.8,142.8,131.7,129.8,115.7,114.2,111.4$, 105.5, 98.7, 68.5, 55.5, 55.4, 52.9, 51.5, 20.6; HRMS (ESI) calcd for $\mathrm{C}_{20} \mathrm{H}_{22} \mathrm{~N}_{2} \mathrm{O}_{4} \mathrm{Br}\left({ }^{79} \mathrm{Br}\right) 433.0757$ $\left[\mathrm{M}+\mathrm{H}^{+}\right]$, found 433.0749 .

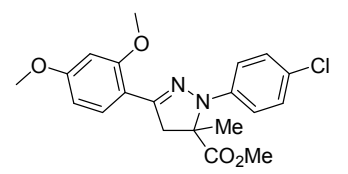

Methyl 1-(4-chlorophenyl)-3-(2,4-dimethoxyphenyl)-5-methyl-4,5-dihydro$1 \mathrm{H}$-pyrazole-5-carboxylate (Table 3, entry 9): ${ }^{1} \mathrm{H} \mathrm{NMR}\left(400 \mathrm{MHz}, \mathrm{CDCl}_{3}\right) \delta$ $7.91(\mathrm{~d}, J=8.8 \mathrm{~Hz}, 2 \mathrm{H}), 7.18(\mathrm{~d}, J=8.8 \mathrm{~Hz}, 2 \mathrm{H}), 6.99(\mathrm{~d}, J=8.8 \mathrm{~Hz}, 2 \mathrm{H}), 6.55$ $(\mathrm{dd}, J=8.8,2.0 \mathrm{~Hz}, 1 \mathrm{H}), 6.46(\mathrm{~d}, J=1.6 \mathrm{~Hz}, 1 \mathrm{H}), 3.85(\mathrm{~s}, 3 \mathrm{H}), 3.83(\mathrm{~s}, 3 \mathrm{H})$, $3.82(\mathrm{~d}, J=17.6 \mathrm{~Hz}, 1 \mathrm{H}), 3.76(\mathrm{~s}, 3 \mathrm{H}), 3.46(\mathrm{~d}, J=17.6 \mathrm{~Hz}, 1 \mathrm{H}), 1.59$ (s, 3H); ${ }^{13} \mathrm{C} \mathrm{NMR}(75.4 \mathrm{MHz}$, $\left.\mathrm{CDCl}_{3}\right) \delta 174.5,161.8,158.7,145.7,142.4,129.8,128.8,124.2,115.4,114.2,105.5,98.7,68.6,55.5$, 55.4, 52.9, 51.5, 20.7; HRMS (ESI) calcd for $\mathrm{C}_{20} \mathrm{H}_{22} \mathrm{~N}_{2} \mathrm{O}_{4} \mathrm{Cl}\left({ }^{35} \mathrm{Cl}\right) 389.1263\left[\mathrm{M}+\mathrm{H}^{+}\right]$, found 389.1263.

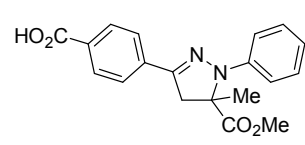

4-(5-(methoxycarbonyl)-5-methyl-1-phenyl-4,5-dihydro-1 $H$-pyrazol-3-yl)ben zoic acid (Table 3, entry 10): ${ }^{1} \mathrm{H}$ NMR $\left(400 \mathrm{MHz}, \mathrm{CDCl}_{3}\right) \delta 8.13(\mathrm{~d}, J=8.4 \mathrm{~Hz}$, $2 \mathrm{H}), 7.78(\mathrm{~d}, J=8.8 \mathrm{~Hz}, 2 \mathrm{H}), 7.28(\mathrm{t}, J=8.8 \mathrm{~Hz}, 2 \mathrm{H}), 7.14(\mathrm{~d}, J=7.6 \mathrm{~Hz}, 2 \mathrm{H})$, $6.93(\mathrm{t}, J=7.2 \mathrm{~Hz}, 1 \mathrm{H}), 3.79(\mathrm{~s}, 3 \mathrm{H}), 3.73(\mathrm{~d}, J=16.8 \mathrm{~Hz}, 1 \mathrm{H}), 3.32(\mathrm{~d}, J=16.8 \mathrm{~Hz}, 1 \mathrm{H}), 1.69$ (s, $3 \mathrm{H}) ;{ }^{13} \mathrm{C} \mathrm{NMR}\left(75.4 \mathrm{MHz}, \mathrm{CDCl}_{3}\right) \delta 173.9,171.4,143.6,142.7,137.5,130.5,129.1,128.6,125.4$, 120.8, 114.8, 69.4, 53.1, 47.7, 21.3; HRMS (ESI) calcd for $\mathrm{C}_{19} \mathrm{H}_{19} \mathrm{~N}_{2} \mathrm{O}_{4} 339.1339\left[\mathrm{M}+\mathrm{H}^{+}\right]$, found 339.1339 .

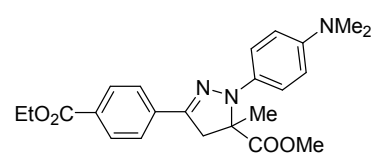

Methyl 1-(4-(dimethylamino)phenyl)-3-(4-(ethoxycarbonyl)phenyl)-5methyl-4,5-dihydro-1 $\boldsymbol{H}$-pyrazole-5-carboxylate (Table 3 , entry 11 ): ${ }^{1} \mathrm{H}$ NMR $\left(300 \mathrm{MHz}, \mathrm{CDCl}_{3}\right) \delta 8.03(\mathrm{~d}, J=8.7 \mathrm{~Hz}, 2 \mathrm{H}), 7.70(\mathrm{~d}, J=8.1 \mathrm{~Hz}, 2 \mathrm{H})$, $7.06(\mathrm{~d}, J=9.0 \mathrm{~Hz}, 2 \mathrm{H}), 6.74(\mathrm{~d}, J=9.0 \mathrm{~Hz}, 2 \mathrm{H}), 4.39(\mathrm{q}, J=7.2 \mathrm{~Hz}, 2 \mathrm{H}), 3.78(\mathrm{~s}, 3 \mathrm{H}), 3.69(\mathrm{~d}, J=$ $16.8 \mathrm{~Hz}, 1 \mathrm{H}), 3.26(\mathrm{~d}, J=16.8 \mathrm{~Hz}, 1 \mathrm{H}), 2.91(\mathrm{~s}, 6 \mathrm{H}), 1.56(\mathrm{~s}, 3 \mathrm{H}), 1.41(\mathrm{t}, J=6.9 \mathrm{~Hz}, 3 \mathrm{H}) ;{ }^{13} \mathrm{C} \mathrm{NMR}$ $\left(75.4 \mathrm{MHz}, \mathrm{CDCl}_{3}\right) \delta 174.0,166.4,143.3,136.9,129.8,129.6,125.1,118.5,114.0,70.7,61.0,52.9$, 47.1, 41.4, 21.3, 14.3; HRMS (ESI) calcd for $\mathrm{C}_{23} \mathrm{H}_{28} \mathrm{~N}_{3} \mathrm{O}_{4} 410.2074\left[\mathrm{M}+\mathrm{H}^{+}\right]$, found 410.2069.

Methyl 5-methyl-1-phenyl-3-(pyridin-2-yl)-4,5-dihydro-1 $H$-pyrazole-5carboxylate (Table 3 , entry 12$):{ }^{1} \mathrm{H}$ NMR $\left(500 \mathrm{MHz}, \mathrm{CDCl}_{3}\right) \delta 8.55(\mathrm{dd}, J=4.5,1.0$ 
$\mathrm{Hz}, 1 \mathrm{H}), 8.11(\mathrm{~d}, J=7.5 \mathrm{~Hz}, 1 \mathrm{H}), 7.70(\mathrm{~d}-\mathrm{t}, J=8.0,2.0 \mathrm{~Hz}, 1 \mathrm{H}), 7.29-7.26(\mathrm{~m}, 2 \mathrm{H}), 7.20$ (d-q, $J=$ $5.0,1.0 \mathrm{~Hz}, 1 \mathrm{H}), 7.13(\mathrm{dd}, J=8.5,1.0 \mathrm{~Hz}, 2 \mathrm{H}), 6.91(\mathrm{t}, J=7.0 \mathrm{~Hz}, 1 \mathrm{H}), 3.82(\mathrm{~d}, J=17.5 \mathrm{~Hz}, 1 \mathrm{H})$, $3.76(\mathrm{~s}, 3 \mathrm{H}), 3.50(\mathrm{~d}, J=18.0 \mathrm{~Hz}, 1 \mathrm{H}), 1.68(\mathrm{~s}, 3 \mathrm{H}) ;{ }^{13} \mathrm{C} \mathrm{NMR}\left(75.4 \mathrm{MHz}, \mathrm{CDCl}_{3}\right) \delta 174.0,165.0$, 149.0, 142.8, 136.0, 129.1, 122.8, 120.7, 120.5, 114.6, 69.2, 53.0, 48.0, 21.2; HRMS (ESI) calcd for $\mathrm{C}_{17} \mathrm{H}_{18} \mathrm{~N}_{3} \mathrm{O}_{2} 296.1394\left[\mathrm{M}+\mathrm{H}^{+}\right]$, found 296.1390.

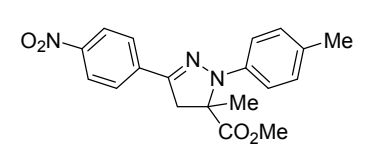

Methyl 5-methyl-3-(4-nitrophenyl)-1-p-tolyl-4,5-dihydro-1 $H$-pyrazole-5carboxylate (Table 3 , entry 13$):{ }^{1} \mathrm{H}$ NMR $\left(300 \mathrm{MHz}, \mathrm{CDCl}_{3}\right) \delta 8.23(\mathrm{~d}, J=9.0$ $\mathrm{Hz}, 2 \mathrm{H}), 7.78(\mathrm{~d}, J=8.7 \mathrm{~Hz}, 2 \mathrm{H}), 7.10(\mathrm{~d}, J=8.4 \mathrm{~Hz}, 2 \mathrm{H}), 7.03(\mathrm{~d}, J=8.7 \mathrm{~Hz}$, 2H), $3.79(\mathrm{~s}, 3 \mathrm{H}), 3.71(\mathrm{~d}, J=16.5 \mathrm{~Hz}, 1 \mathrm{H}), 3.29(\mathrm{~d}, J=16.8 \mathrm{~Hz}, 1 \mathrm{H}), 2.30(\mathrm{~s}, 3 \mathrm{H}), 1.67(\mathrm{~s}, 3 \mathrm{H}) ;{ }^{13} \mathrm{C}$ $\operatorname{NMR}\left(75.4 \mathrm{MHz}, \mathrm{CDCl}_{3}\right) \delta 173.6,147.0,141.9,140.0,138.7,131.0,129.7,125.7,124.0,115.3,70.0$, 53.1, 47.3, 21.4, 20.6; HRMS (EI) calcd for $\mathrm{C}_{19} \mathrm{H}_{19} \mathrm{~N}_{3} \mathrm{O}_{4} 353.1370\left[\mathrm{M}^{+}\right]$, found 353.1381.

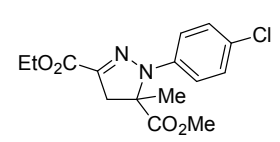

3-Ethyl 5-methyl 1-(4-chlorophenyl)-5-methyl-4,5-dihydro-1 $\boldsymbol{H}$-pyrazole-3,5dicarboxylate (Table 3 , entry 14$):{ }^{1} \mathrm{H}$ NMR $\left(400 \mathrm{MHz}, \mathrm{CDCl}_{3}\right) \delta 7.22(\mathrm{~d}, J=8.8$ $\mathrm{Hz}, 2 \mathrm{H}), 7.05(\mathrm{~d}, J=9.2 \mathrm{~Hz}, 2 \mathrm{H}), 4.35$ (q, $J=7.2 \mathrm{~Hz}, 2 \mathrm{H}), 3.77$ (s, 3H), 3.57 (d, $J$ $=18.0 \mathrm{~Hz}, 1 \mathrm{H}), 3.19(\mathrm{~d}, J=18.0 \mathrm{~Hz}, 1 \mathrm{H}), 1.65(\mathrm{~s}, 3 \mathrm{H}), 1.38(\mathrm{t}, J=7.2 \mathrm{~Hz}, 3 \mathrm{H}) ;{ }^{13} \mathrm{C} \mathrm{NMR}(75.4$ $\left.\mathrm{MHz}, \mathrm{CDCl}_{3}\right) \delta 172.7,162.1,140.1,137.3,129.1,127.2,116.7,70.4,61.4,53.2,47.1,21.4,14.3$; HRMS (ESI) calcd for $\mathrm{C}_{15} \mathrm{H}_{17} \mathrm{~N}_{2} \mathrm{O}_{4} \mathrm{ClNa} 347.0769\left[\mathrm{M}+\mathrm{Na}^{+}\right]$, found 347.0763.

\section{Synthesis of tetrazoles 4 and 5, and pyrazoline 6}

\section{4-(2-\{4-[2-(4-Chloro-phenyl)-2H-tetrazol-5-yl]-3-methoxy-phenoxy\}-ethyl)-morpholine (4)} Preparation of the phenylsufonylhydrazone (A): A mixture of 4-hydroxy-

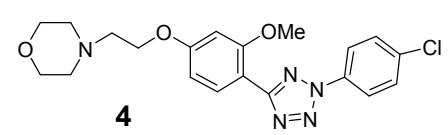
2-methoxybenzaldehyde (5.00 g, $32.8 \mathrm{mmol})$, 4-(2-chloroethyl)morpholine hydrochloride (18.34 g, $98.5 \mathrm{mmol})$, and $\mathrm{K}_{2} \mathrm{CO}_{3}(68.1 \mathrm{~g}$, $492.9 \mathrm{mmol})$ in $65 \mathrm{~mL}$ anhydrous DMF was heated to $50{ }^{\circ} \mathrm{C}$ for $21 \mathrm{hr}$.

The mixture was allowed to cool down to room temperature before water was added, and the resulting mixture was extracted with ethyl acetate. The organic layers were combined, dried over sodium sulfate, and concentrated under the reduced pressure to give the alkylation product as orange oil. To the crude product in $130 \mathrm{~mL}$ EtOH was added benzenesulfonyl hydrazide $(5.6 \mathrm{~g}, 32.8$ $\mathrm{mmol}$ ), and the mixture was stirred for $1.5 \mathrm{hr}$. After dilution with cold water, precipitates from the reaction were collected and dried in vacuo to afford the phenylsufonylhydrazone (A) as a white solid; Preparation of 4-chlorobenzenediazonium salt (B): To an ice-water bath cooled solution of sodium nitrate $(1.38 \mathrm{~g}, 20 \mathrm{mmol})$ in $8 \mathrm{~mL}$ water was added dropwise a solution of 4-chloroaniline $(2.5 \mathrm{~g}, 20$ $\mathrm{mmol}$ ) in $8 \mathrm{~mL} \mathrm{HCl}$ and $32 \mathrm{~mL} \mathrm{50 \%} \mathrm{ethanol,} \mathrm{and} \mathrm{the} \mathrm{mixture} \mathrm{was} \mathrm{stirred} \mathrm{to} \mathrm{give} \mathrm{the} \mathrm{arenediazonium}$ salt (B); Tetrazole 4 formation: Solution B $(20 \mathrm{mmol})$ was added dropwise to a stirred solution of A $(6.8 \mathrm{~g}, 16.4 \mathrm{mmol})$ in $125 \mathrm{~mL}$ pyridine at $-10 \sim-15{ }^{\circ} \mathrm{C}$. The reaction mixture was allowed to warm up to r.t. over $45 \mathrm{~min}$, and the solution was extracted with choloform $(3 \times)$. The organic layers were combined, dried over sodium sulfate, and concentrated under reduced pressure to afford a crude oil. The crude was purified by flash chromatography on a silica gel column using a stepwise gradient of $0.5-2.5 \% \mathrm{MeOH} / \mathrm{CH}_{2} \mathrm{Cl}_{2}$ to afford the tetrazole 4 as a yellow oil $(2.8 \mathrm{~g}, 42 \%):{ }^{1} \mathrm{H}$ NMR $(500 \mathrm{MHz}$, $\left.\mathrm{CDCl}_{3}\right) \delta 8.14(\mathrm{~d}, J=9 \mathrm{~Hz}, 2 \mathrm{H}), 7.99(\mathrm{~d}, J=9 \mathrm{~Hz}, 1 \mathrm{H}), 7.52(\mathrm{~d}, J=9 \mathrm{~Hz}, 2 \mathrm{H}), 6.64(\mathrm{~m}, 2 \mathrm{H}), 4.20$ (t, 
$J=5.5 \mathrm{~Hz}, 2 \mathrm{H}), 3.95(\mathrm{~s}, 3 \mathrm{H}), 3.76(\mathrm{t}, J=4.5 \mathrm{~Hz}, 4 \mathrm{H}), 2.86(\mathrm{t}, J=5.5 \mathrm{~Hz}, 2 \mathrm{H}), 2.63(\mathrm{bm}, 4 \mathrm{H}) ;{ }^{13} \mathrm{C}$ $\operatorname{NMR}\left(75.4 \mathrm{MHz}, \mathrm{CDCl}_{3}\right) \delta 53.9,55.8,57.3,65.7,66.6,99.6,105.5,108.9,120.8,129.5,131.5$, 134.9, 135.2, 158.8, 161.7, 163.3; HRMS (ESI) calcd for $\mathrm{C}_{20} \mathrm{H}_{23} \mathrm{~N}_{5} \mathrm{O}_{3} \mathrm{Cl} 416.1484\left[\mathrm{M}+\mathrm{H}^{+}\right]$, found 416.1483 .

\section{4-(2-\{4-[2-(4-Chloro-phenyl)-2H-tetrazol-5-yl]-3-isopropoxy-phenoxy\}-ethyl)-morpholine (5)}

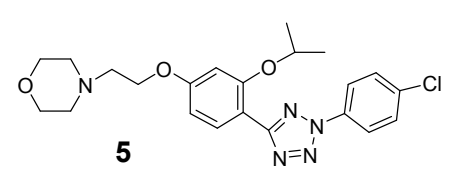

Following a reported procedure, ${ }^{2}$ a solution of $\mathrm{BBr}_{3}(95.6 \mathrm{mg}, 0.38$ mmol, dissolved in $5 \mathrm{ml} \mathrm{CH}_{2} \mathrm{Cl}_{2}$ ) was added drop-wise to a solution of 4 (264.4 $\mathrm{mg}, 0.65 \mathrm{mmol}$ ) in $\mathrm{CH}_{2} \mathrm{Cl}_{2}$ at $-78{ }^{\circ} \mathrm{C}$ over $5 \mathrm{hr}$, and the reaction was allowed to warm up to r.t. overnight. The isolated crude product was added 2-iodopropane (23 mg, $1.3 \mathrm{mmol}$ ) and $\mathrm{K}_{2} \mathrm{CO}_{3}(38.7 \mathrm{mg}, 2.8 \mathrm{mmol}$ ) in DMF, and the mixture was heated at $50{ }^{\circ} \mathrm{C}$ for $23 \mathrm{~h}$. Water was then added and the aqueous solution was extracted with ethyl acetate. The organic layers were combined, dried over sodium sulfate, and concentrated under the reduced pressure. The residue was purified by flash chromatography on a silica gel column using a stepwise gradient of $0.5-1.5 \% \mathrm{MeOH} / \mathrm{CH}_{2} \mathrm{Cl}_{2}$ to afford the tetrazole 5 as yellow oil (98 mg, 40\%): ${ }^{1} \mathrm{H}$ NMR $\left(500 \mathrm{MHz}, \mathrm{CDCl}_{3}\right) \delta 8.14(\mathrm{~d}, J=9 \mathrm{~Hz}, 2 \mathrm{H}), 8.0(\mathrm{~d}, J=8 \mathrm{~Hz}, 1 \mathrm{H}), 7,53(\mathrm{~d}, J$ $=8.5 \mathrm{~Hz}, 2 \mathrm{H}), 6.64(\mathrm{~d}, J=2.5 \mathrm{~Hz}, 1 \mathrm{H}), 6.63(\mathrm{dd}, J=2.5,9.2 \mathrm{~Hz}, 1 \mathrm{H}), 4.63(\mathrm{~m}, 1 \mathrm{H}), 4,17(\mathrm{t}, J=6.0$ $\mathrm{Hz}, 2 \mathrm{H}), 3.75(\mathrm{t}, J=4.5 \mathrm{~Hz}, 4 \mathrm{H}), 2.80(\mathrm{t}, J=5.8 \mathrm{~Hz}, 2 \mathrm{H}), 2.60(\mathrm{t}, J=4.3 \mathrm{~Hz}, 4 \mathrm{H}), 1.41(\mathrm{~d}, J=6.5$ $\mathrm{Hz}, 6 \mathrm{H}) ;{ }^{13} \mathrm{C} \mathrm{NMR}\left(75.4 \mathrm{MHz}, \mathrm{CDCl}_{3}\right) \delta 164.1,162.1,158.0,136.0,135.5,132.2,130.3,121.3$, 111.1, 106.7,103.2, 72.3, 67.4, 66.4, 58.0, 54.6, 22.6; HRMS (ESI) calcd for $\mathrm{C}_{22} \mathrm{H}_{27} \mathrm{~N}_{5} \mathrm{O}_{3} \mathrm{Cl} 444.1797$ $\left[\mathrm{M}+\mathrm{H}^{+}\right]$, found 444.1808 .

\section{4-(2-\{4-[1,5-Bis-(4-chloro-phenyl)-4,5-dihydro-1H-pyrazol-3-yl]-3-isopropoxy-phenoxy\}-ethyl)-} morpholine (6)

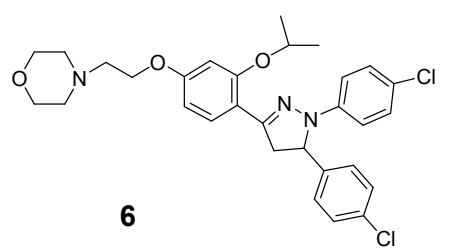

A stirred solution of 5 (36.2 $\mathrm{mg}, 0.08 \mathrm{mmol})$ and 4-chlorostyrene (13.5 $\mathrm{mg}, 0.1 \mathrm{mmol}$ ) in $7 \mathrm{~mL}$ benzene was irradiated with a hand-held $302 \mathrm{~nm}$ UV lamp for $4.5 \mathrm{~h}$. The solvent was then removed under the reduced pressure to give the crude product, which was subsequently purified by flash chromatography on a silica gel column using a stepwise gradient of $0.5-2.5 \% \mathrm{MeOH} / \mathrm{CH}_{2} \mathrm{Cl}_{2}$ to afford the desired pyrazoline product $6(45.1 \mathrm{mg}, 54 \%):{ }^{1} \mathrm{H} \mathrm{NMR}$ $\left(500 \mathrm{MHz}, \mathrm{CDCl}_{3}\right) \delta 7.91(\mathrm{~d}, J=8.5 \mathrm{~Hz}, 1 \mathrm{H}), 7.31(\mathrm{~d}, J=8 \mathrm{~Hz}, 2 \mathrm{H}), 7.24(\mathrm{~d}, J=8.5 \mathrm{~Hz}, 2 \mathrm{H}), 7.11(\mathrm{~d}$, $J=9 \mathrm{~Hz}, 2 \mathrm{H}), 6.93(\mathrm{~d}, J=9 \mathrm{~Hz}, 2 \mathrm{H}), 6.54(\mathrm{dd}, J=2.25,8.75 \mathrm{~Hz}, 1 \mathrm{H}), 6.47(\mathrm{~d}, J=2.5 \mathrm{~Hz}, 1 \mathrm{H}), 5.12$ $(\mathrm{dd}, J=7,12 \mathrm{~Hz}, 1 \mathrm{H}), 4.56(\mathrm{~m}, 1 \mathrm{H}), 4.14(\mathrm{t}, J=5.75 \mathrm{~Hz}, 2 \mathrm{H}), 3.98(\mathrm{dd}, J=18,12 \mathrm{~Hz}, 1 \mathrm{H}), 3.77(\mathrm{t}$, $J=4.8 \mathrm{~Hz}, 4 \mathrm{H}), 3.24(\mathrm{dd}, J=18,7.0 \mathrm{~Hz}, 1 \mathrm{H}), 2.83(\mathrm{t}, J=5.5 \mathrm{~Hz}, 2 \mathrm{H}), 2.60(\mathrm{t}, J=4.5 \mathrm{~Hz}, 4 \mathrm{H}), 1.33$ $(\mathrm{d}, J=6.0 \mathrm{~Hz}, 3 \mathrm{H}), 1.29(\mathrm{~d}, J=6.0 \mathrm{~Hz}, 3 \mathrm{~Hz}) ;{ }^{13} \mathrm{C} \mathrm{NMR}\left(75.4 \mathrm{MHz}, \mathrm{CDCl}_{3}\right) \delta 161.2,157.4,148.2$, 144.2, 141.6, 133.7, 130.4, 129.7, 129.2, 127.9, 123.9, 116.1, 114.8, 106.5, 101.5, 70.9, 67.4, 66.3, 64.3, 58.1, 47.5, 22.6, 22.5; HRMS (ESI) calcd for $\mathrm{C}_{30} \mathrm{H}_{34} \mathrm{Cl}_{2} \mathrm{~N}_{3} \mathrm{O}_{3} 554.1972\left[\mathrm{M}+\mathrm{H}^{+}\right]$, found 554.1954 .

\section{Reference:}

(1) (a) Ito, S.; Tanaka, Y.; Kakehi, A.; Kondo, K. Bull. Chem. Soc. Jpn. 1976, 49, 1920-1923. (b) Ito, S.; Tanaka, Y.; Kakehi, A. Bull. Chem. Soc. Jpn. 1976, 49, 762-766.

(2) Stauffer, S.; Coletta, C.; Tedesco, R.; Nishiguchi, G..; Carlson, K.; Sun, J.; Katzenellenbogen, B. S.; Katzenellenbogen, J. A. J. Med. Chem. 2000, 43, 4934-4947. 

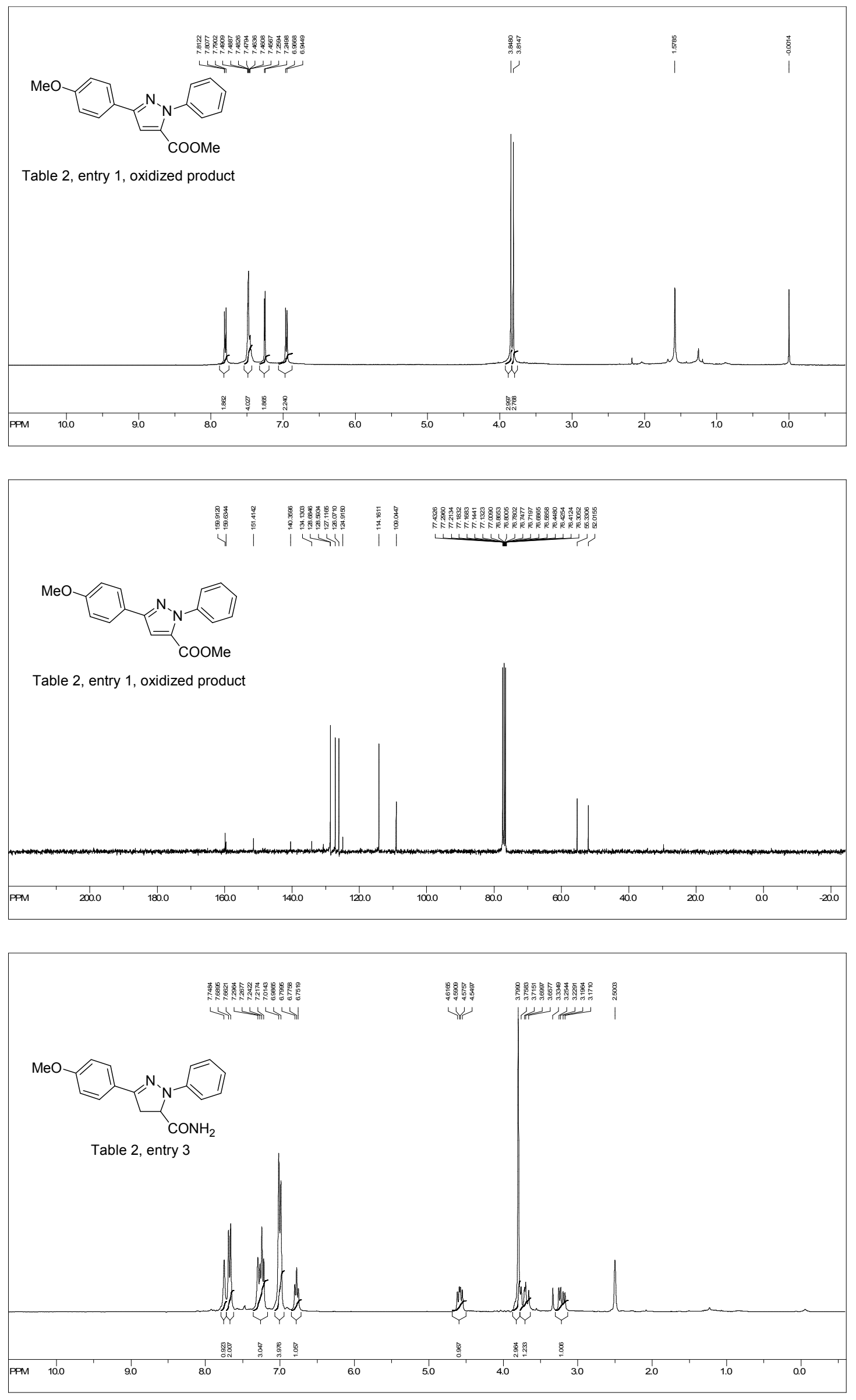

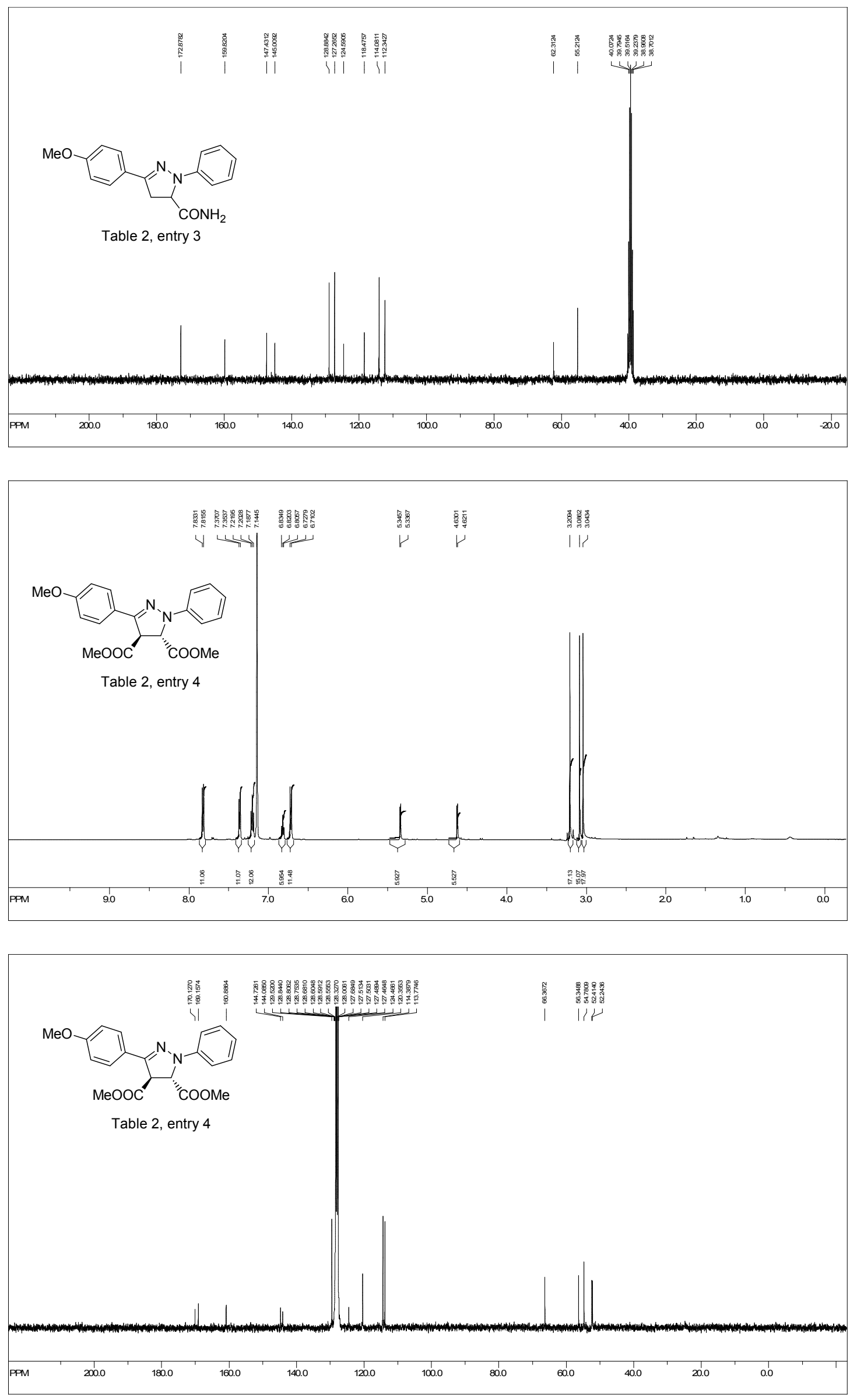

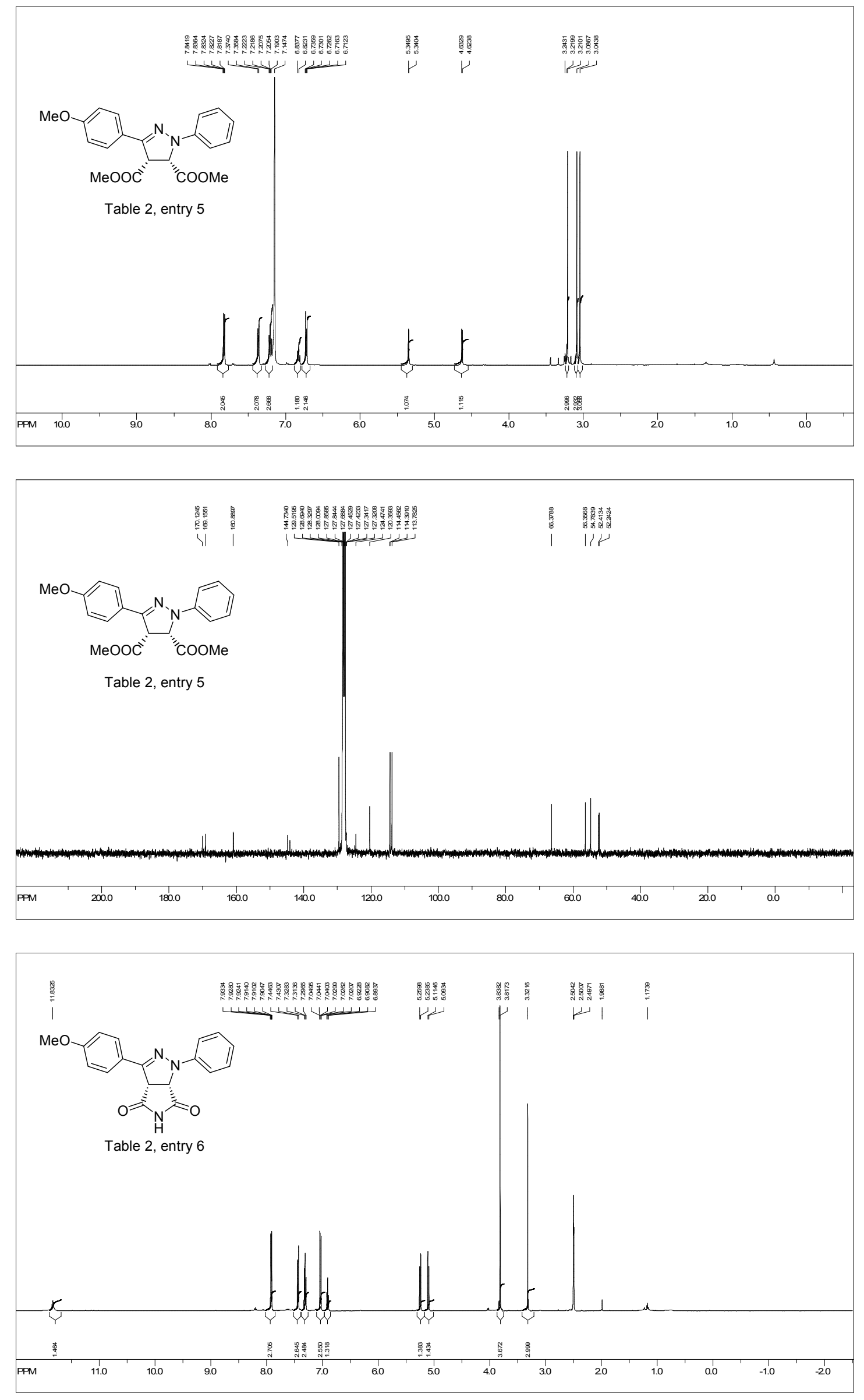

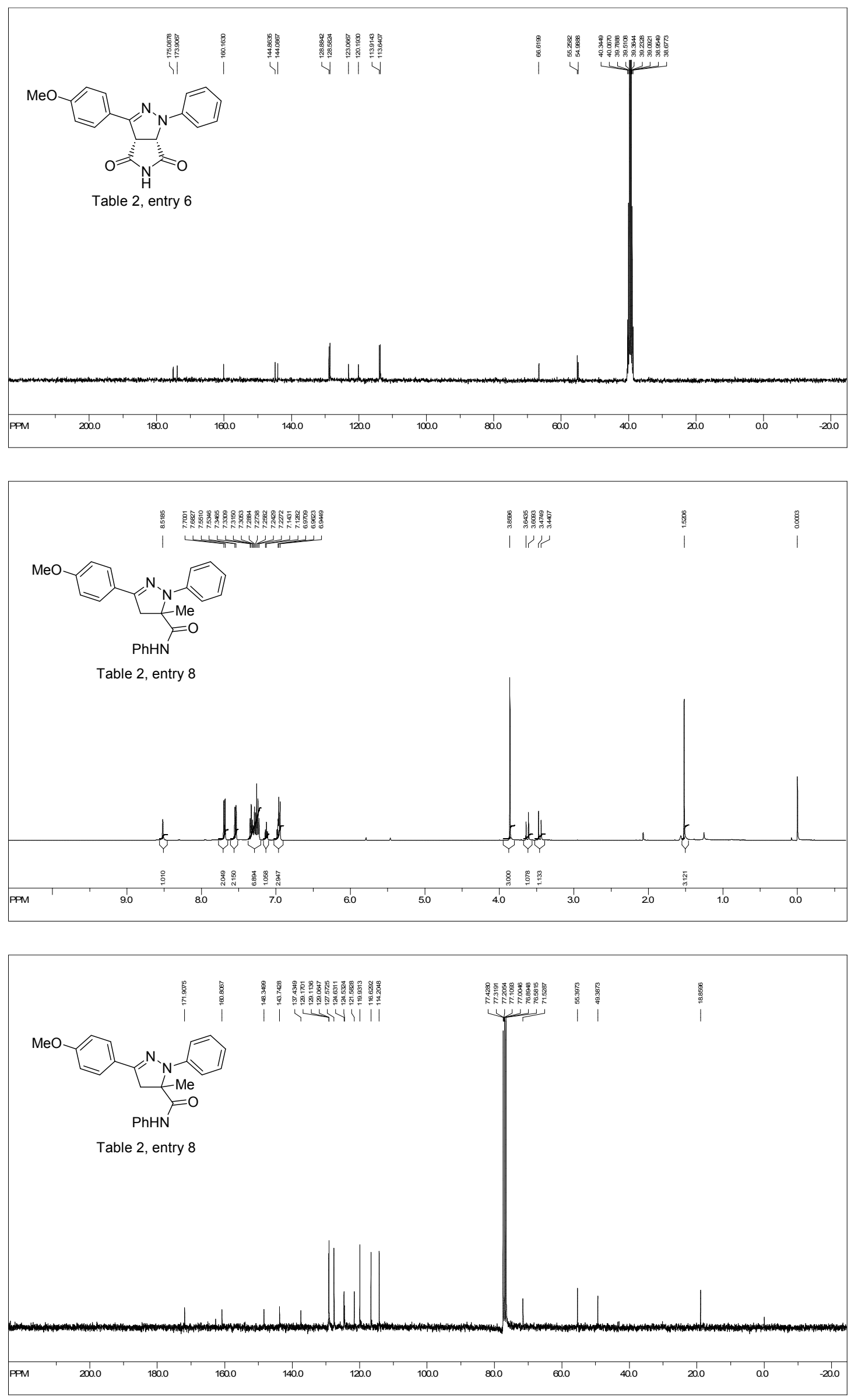

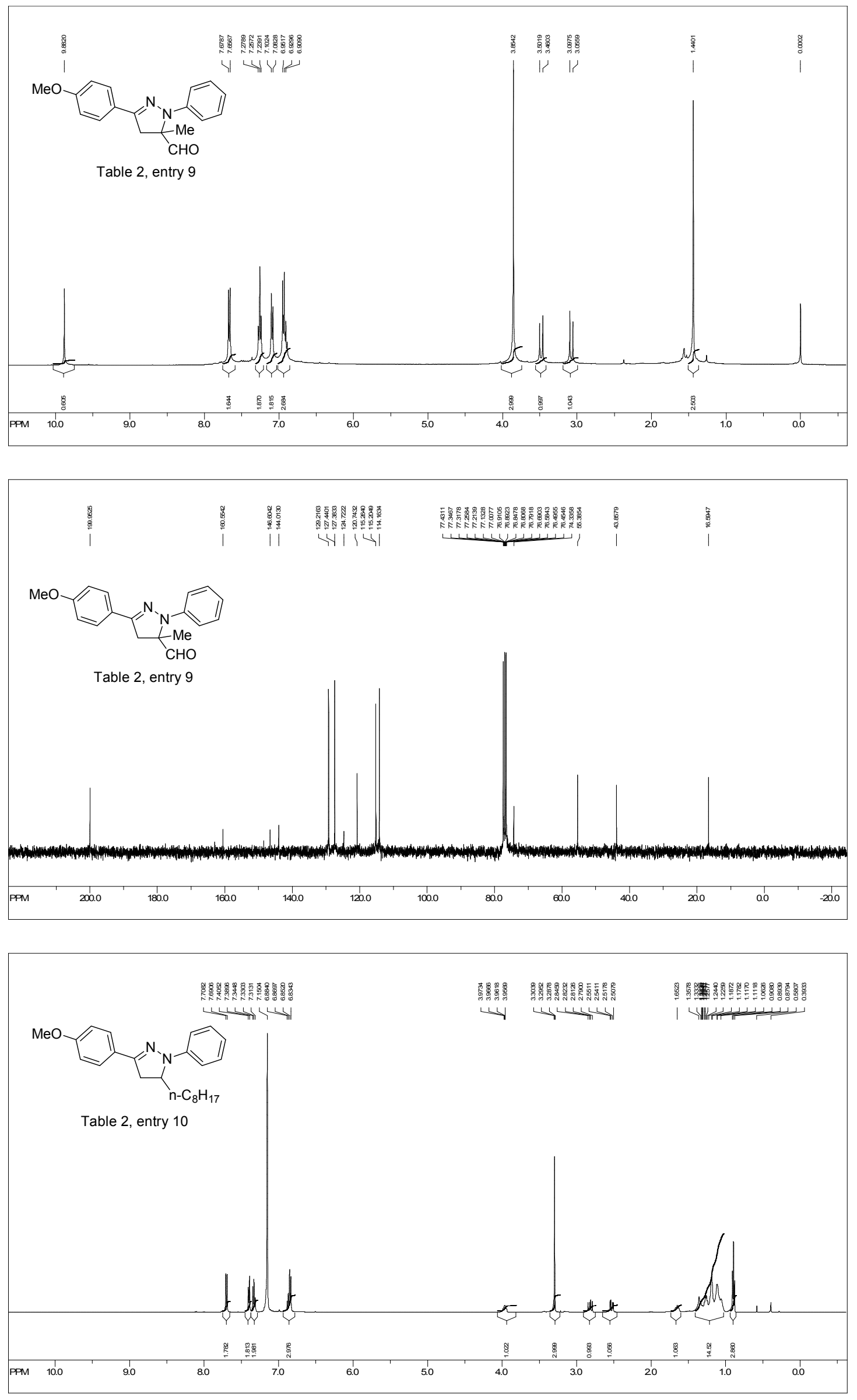

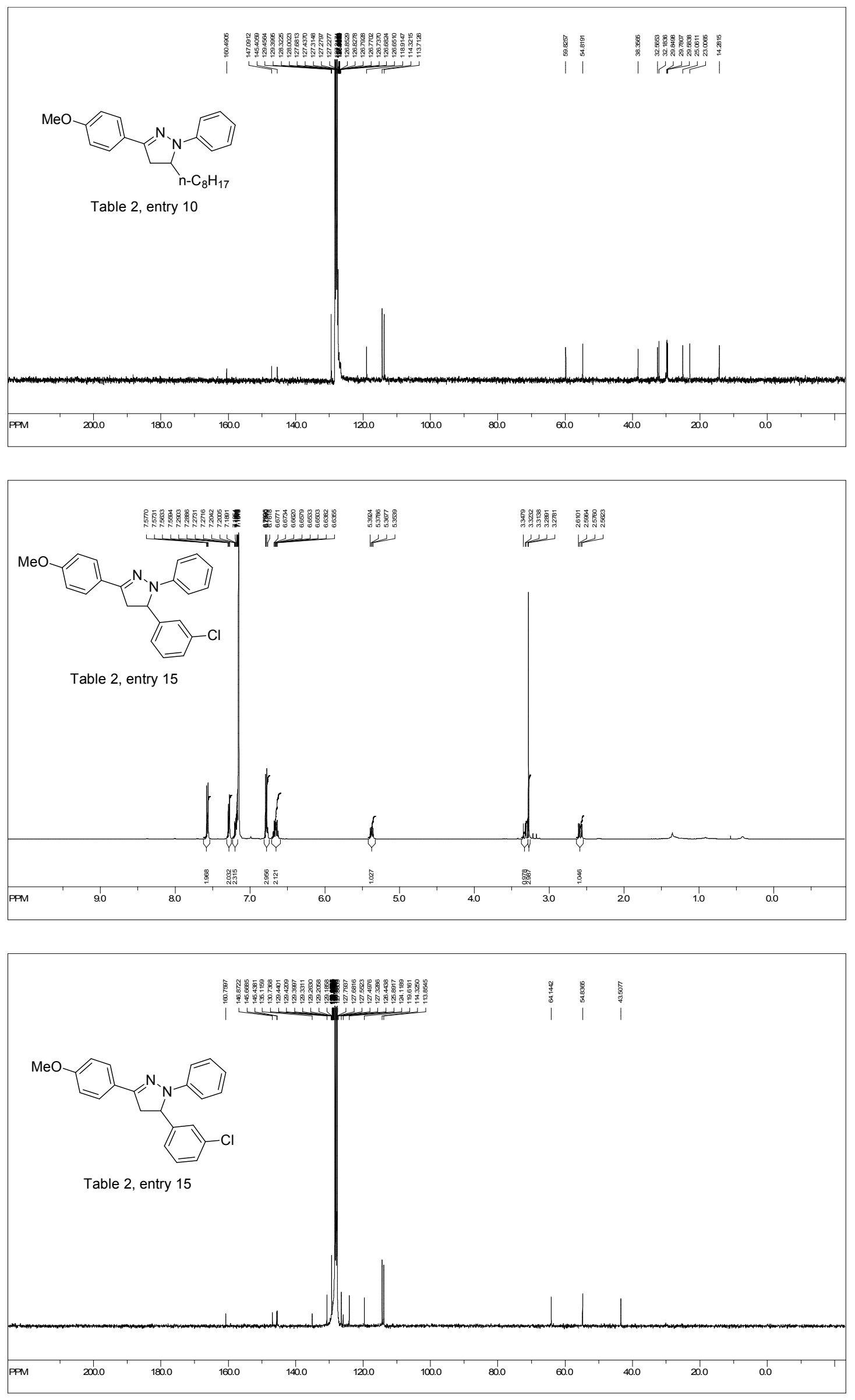

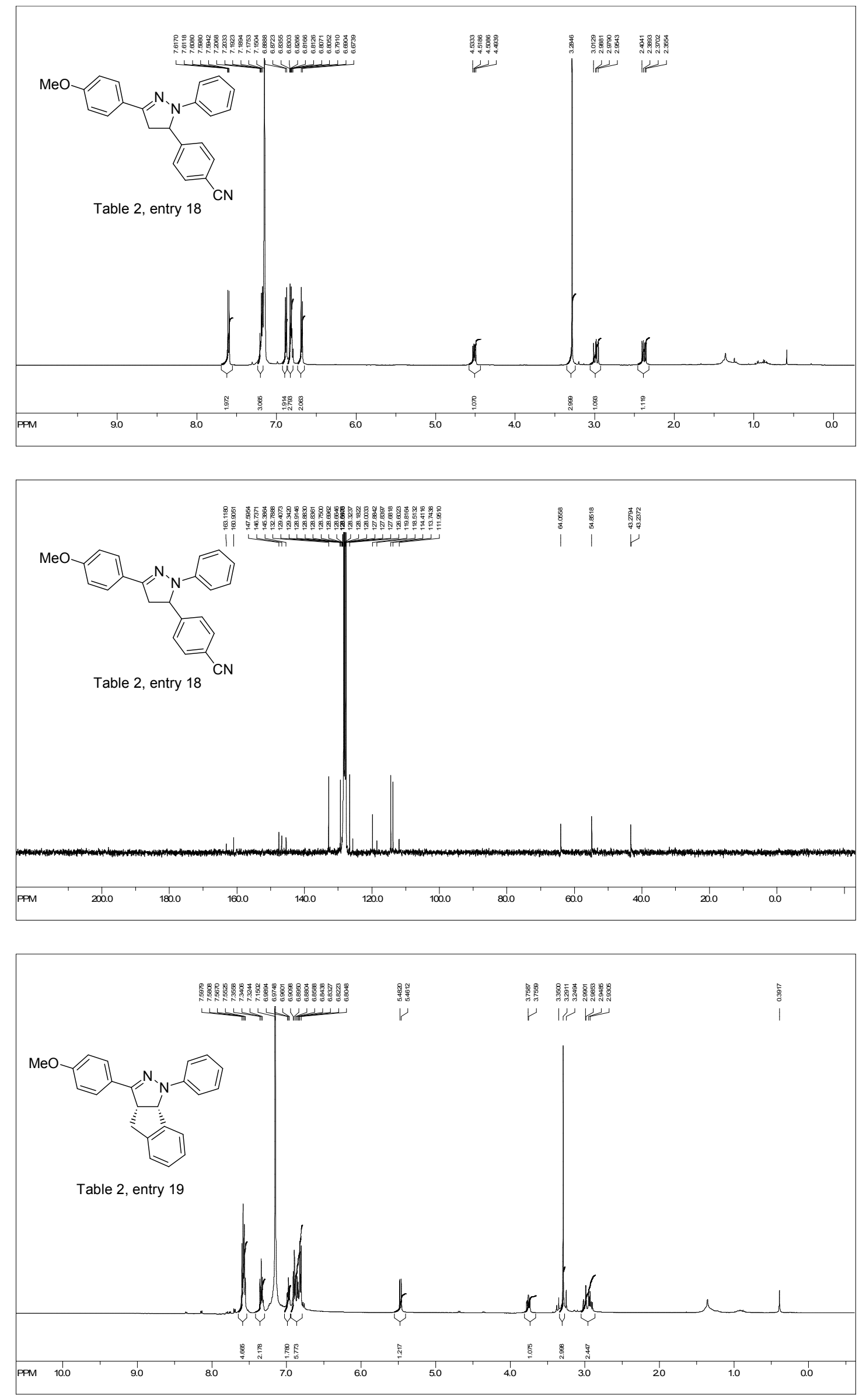

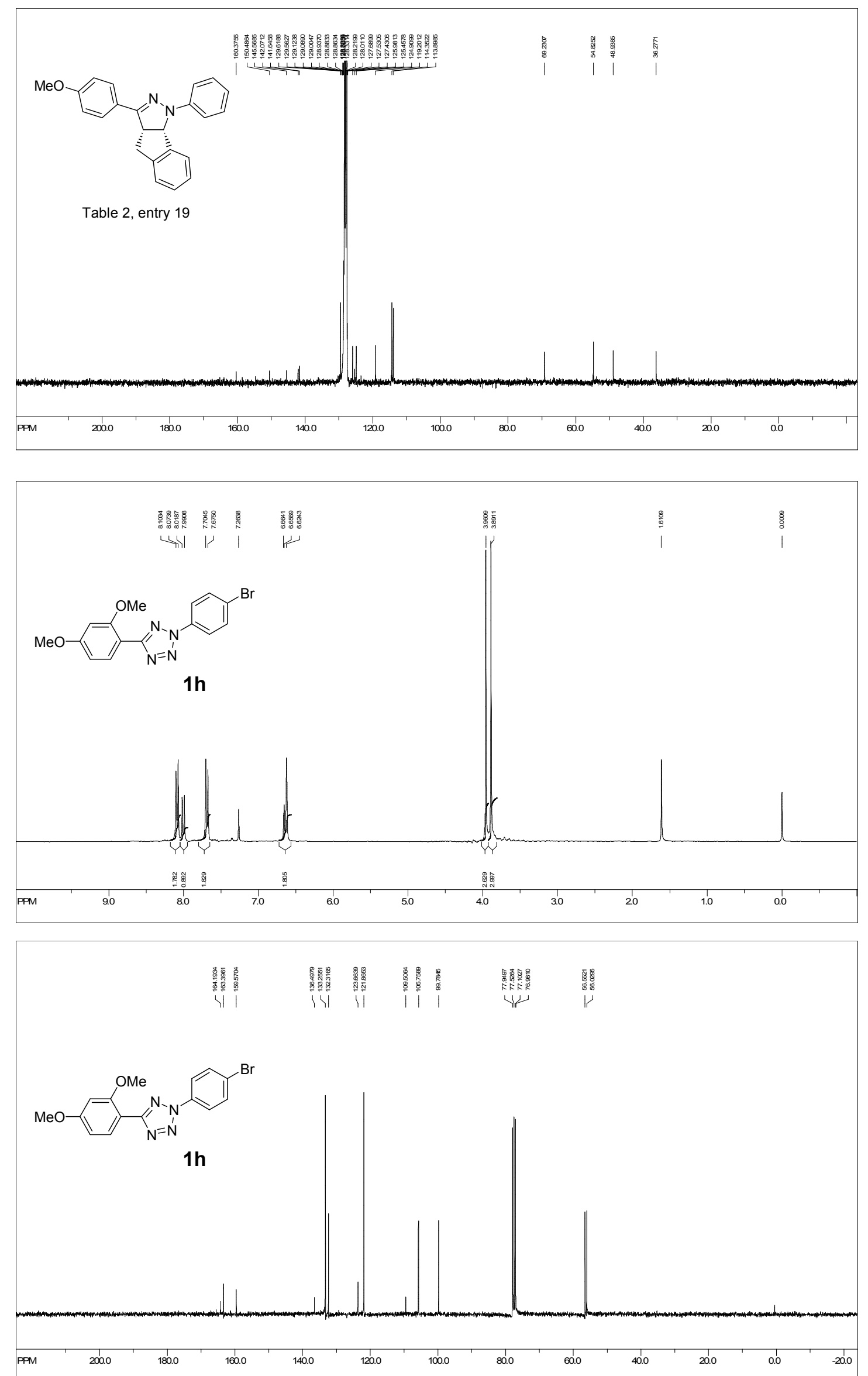

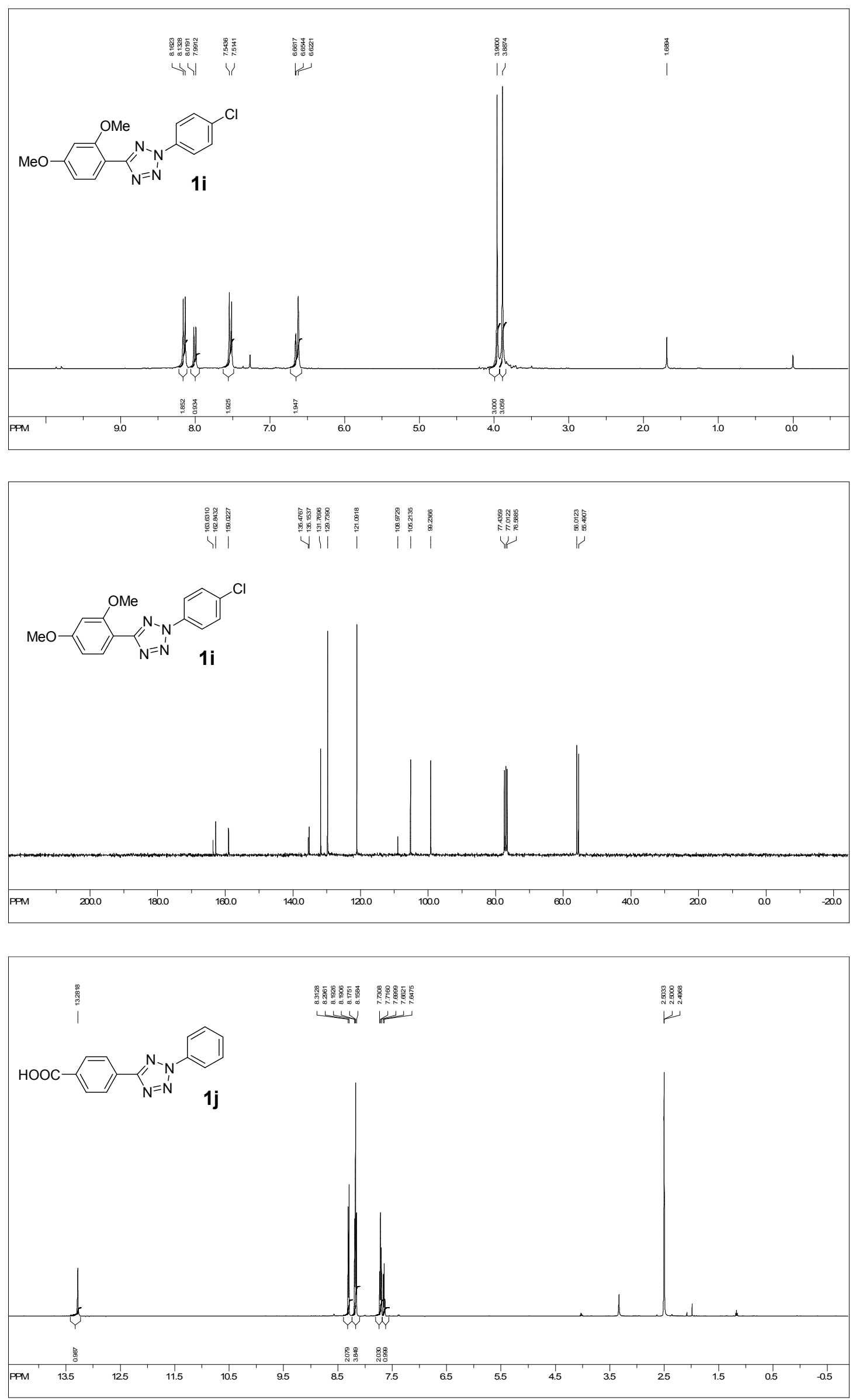


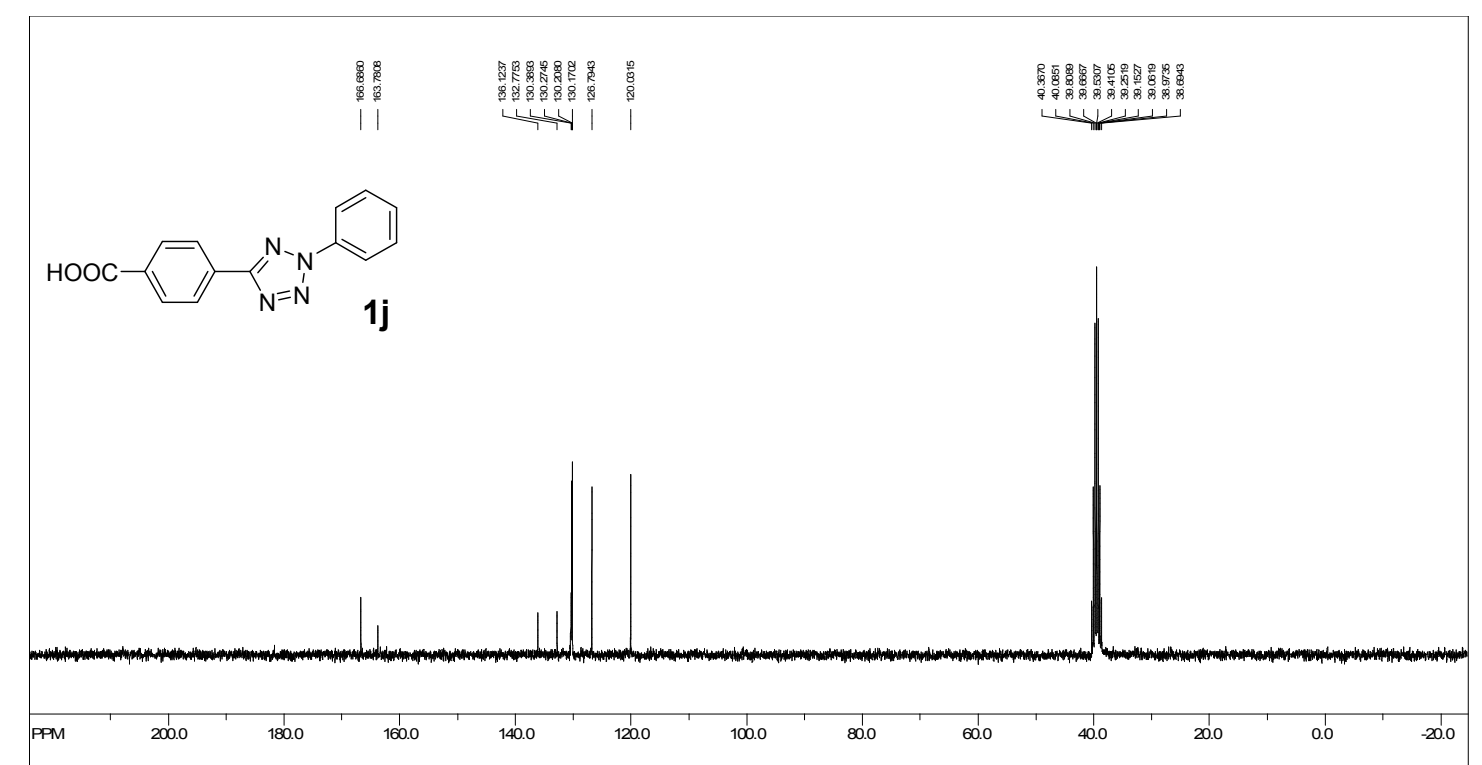

SpinWorks 25: wy2-291-04112007-H

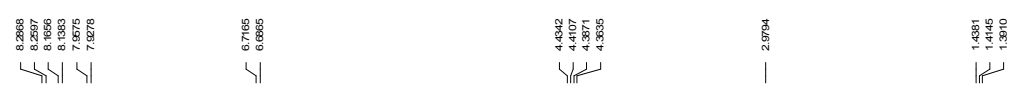<smiles>CCOC(=O)c1ccc(-c2nnn(-c3ccc(N(C)C)cc3)n2)cc1</smiles>
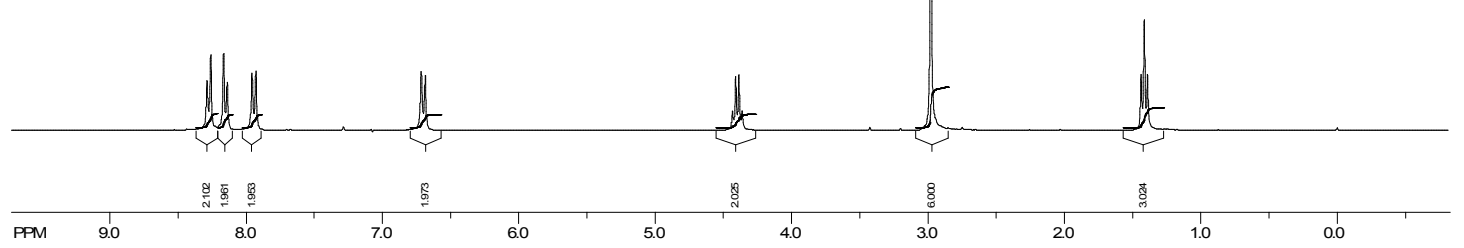

SpinWorks 25: wy2-291-04112007-C13

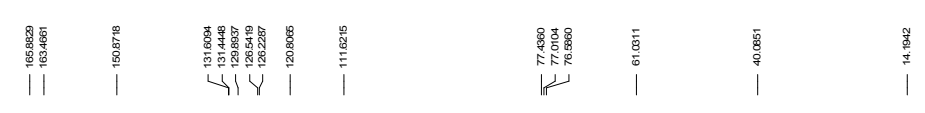<smiles>COC(=O)c1ccc(-c2nnn(-c3ccc(N(C)C)cc3)n2)cc1</smiles>

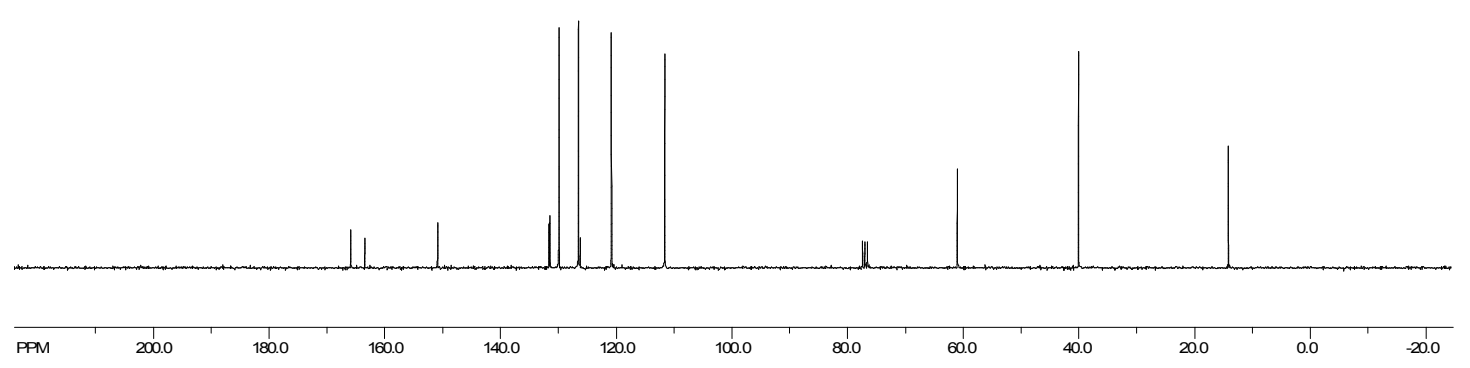



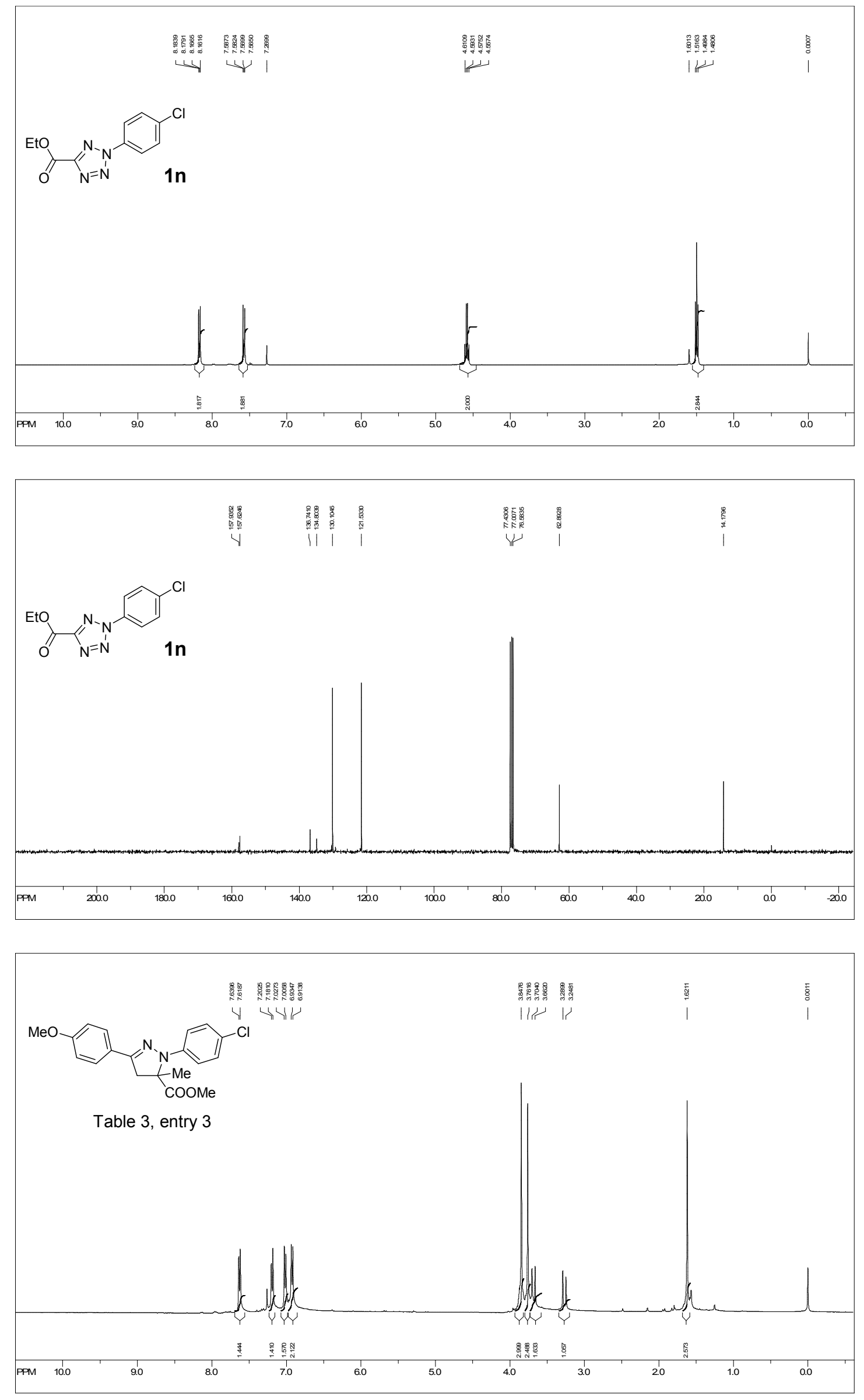

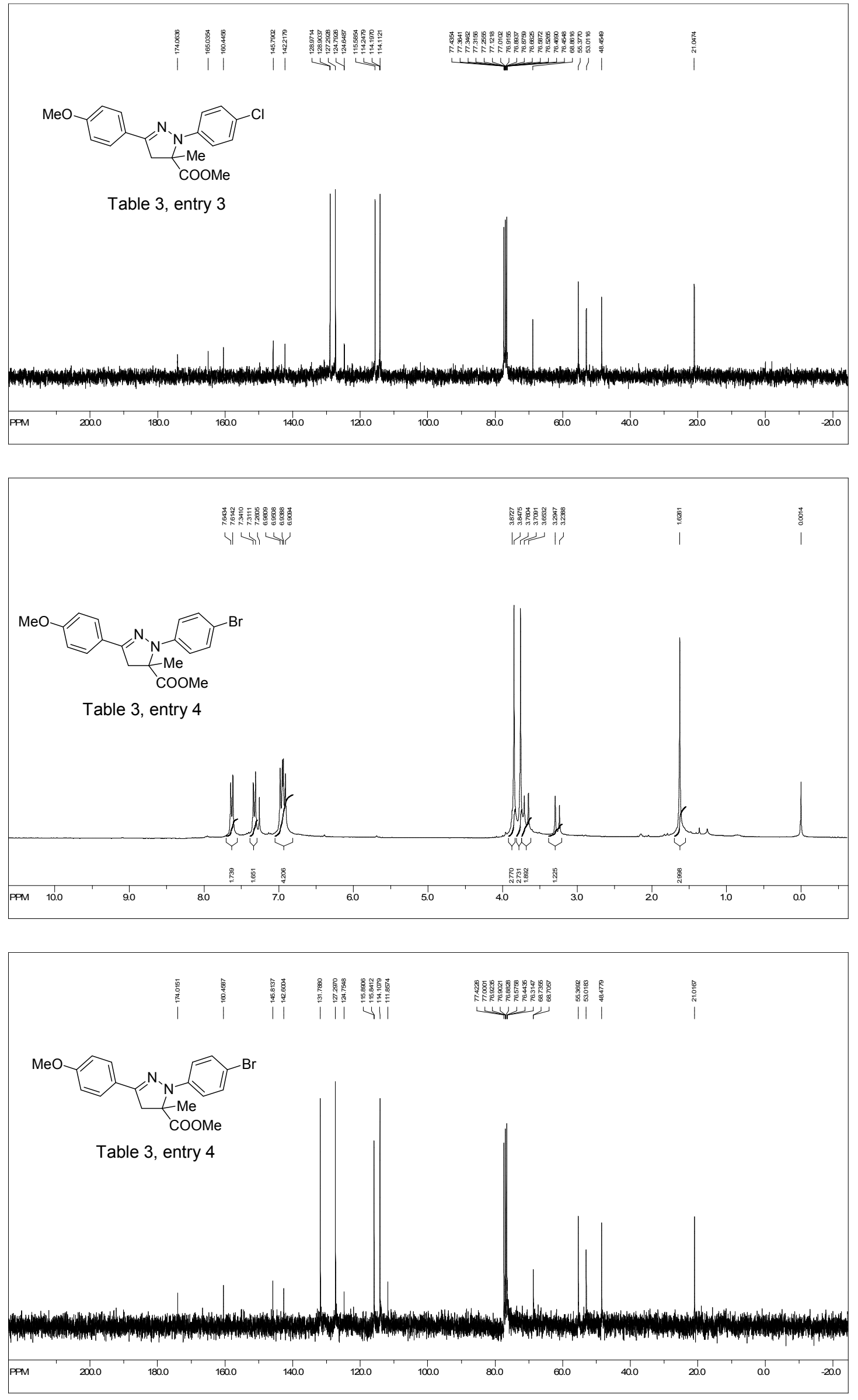

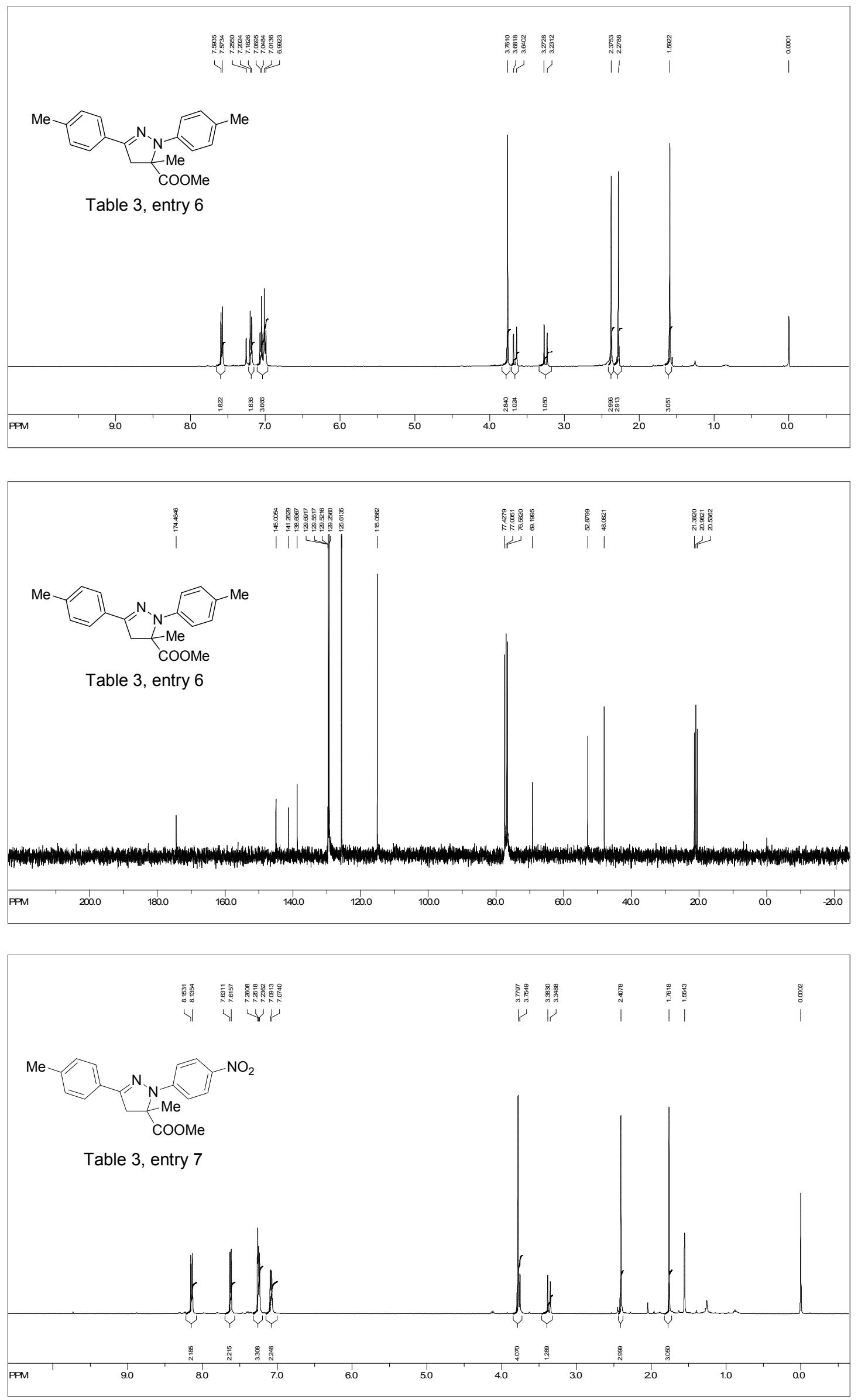

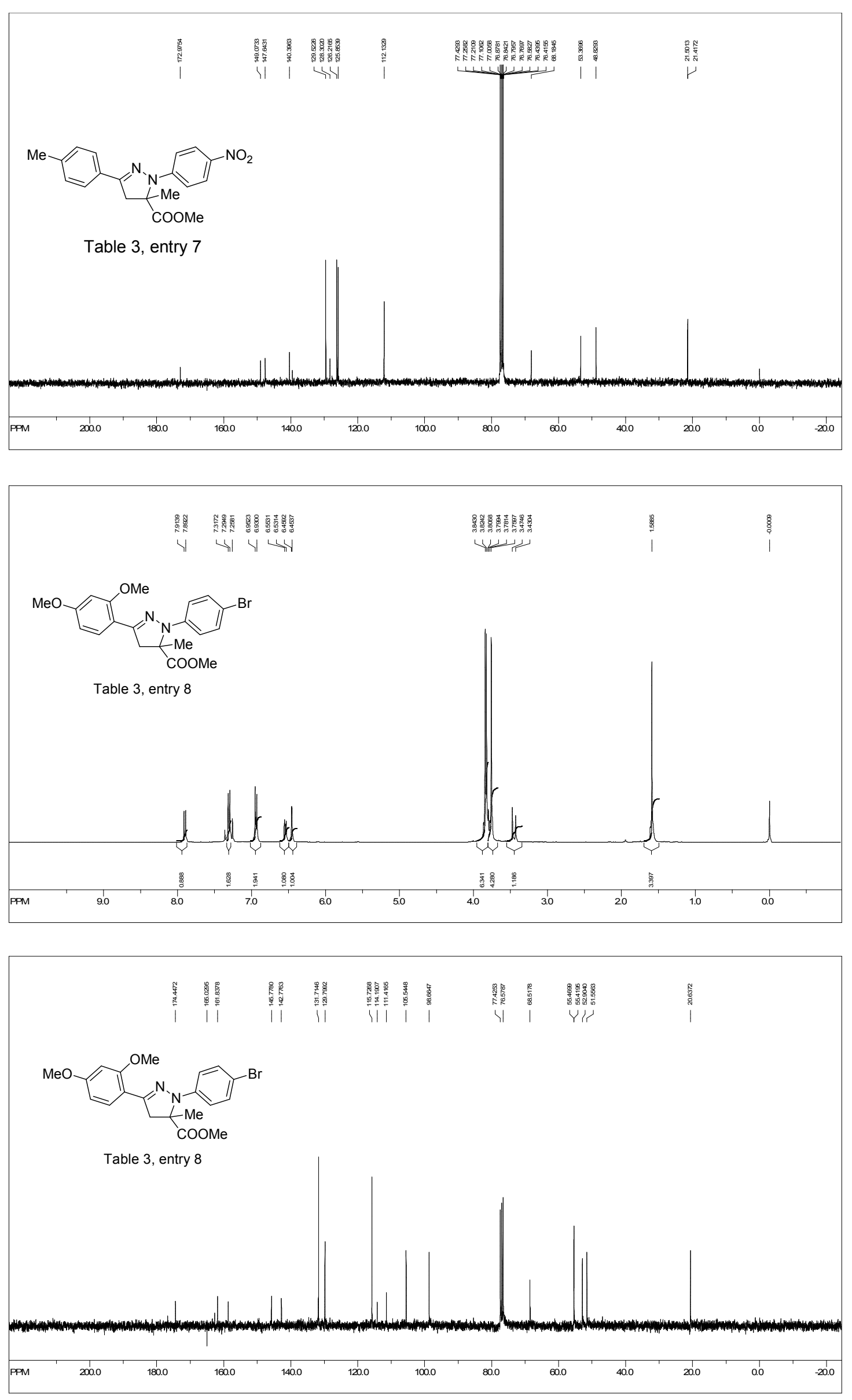

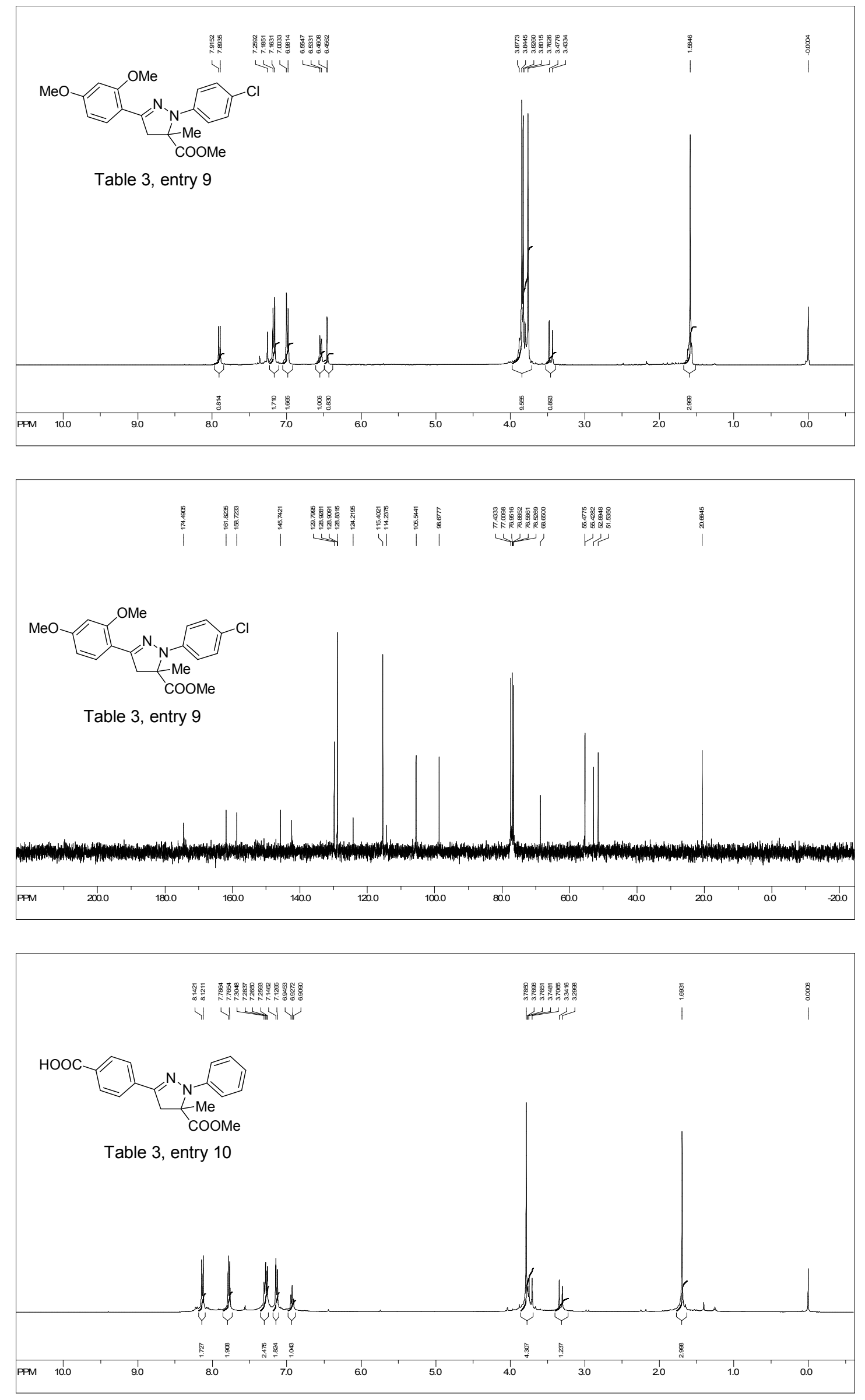

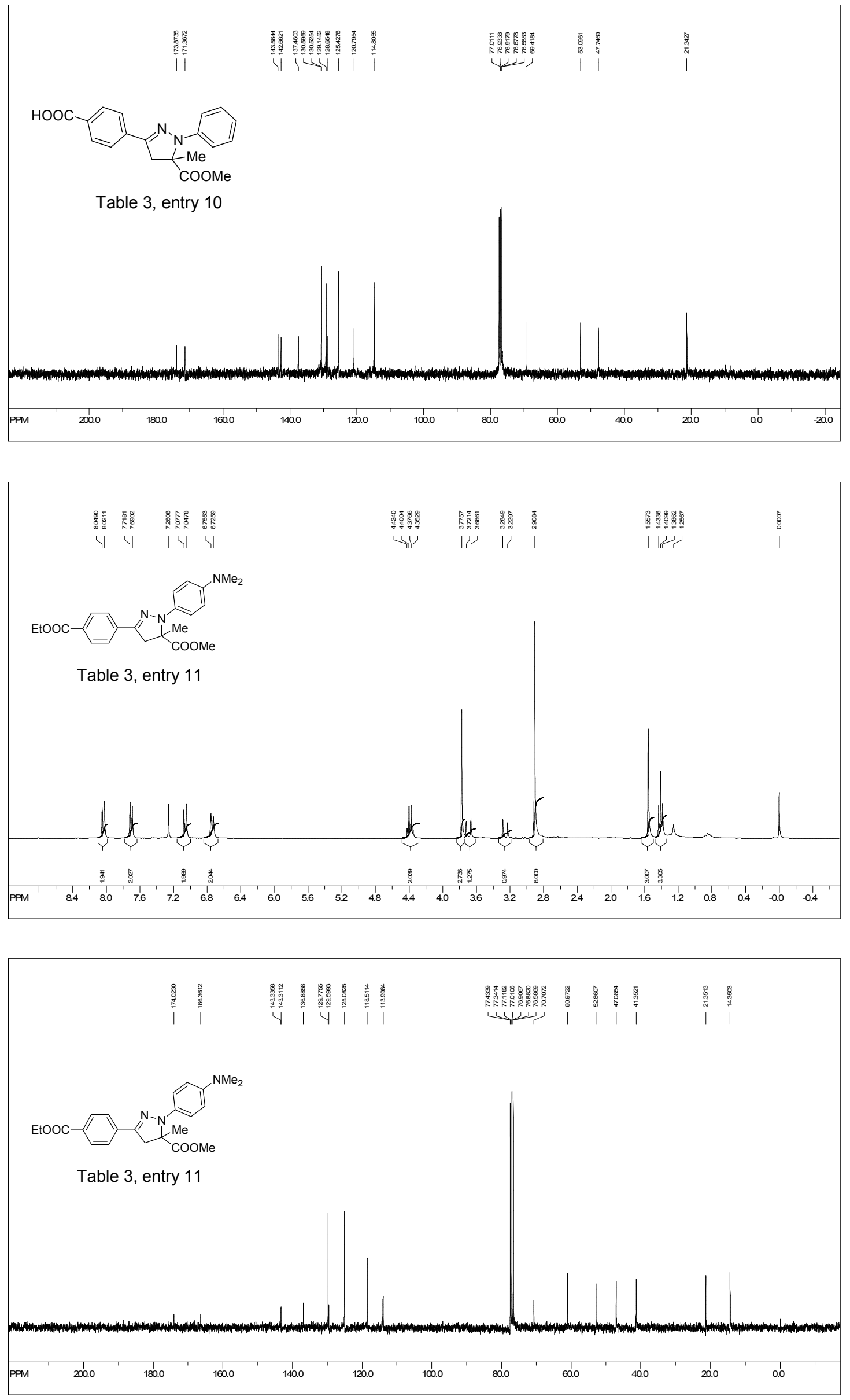

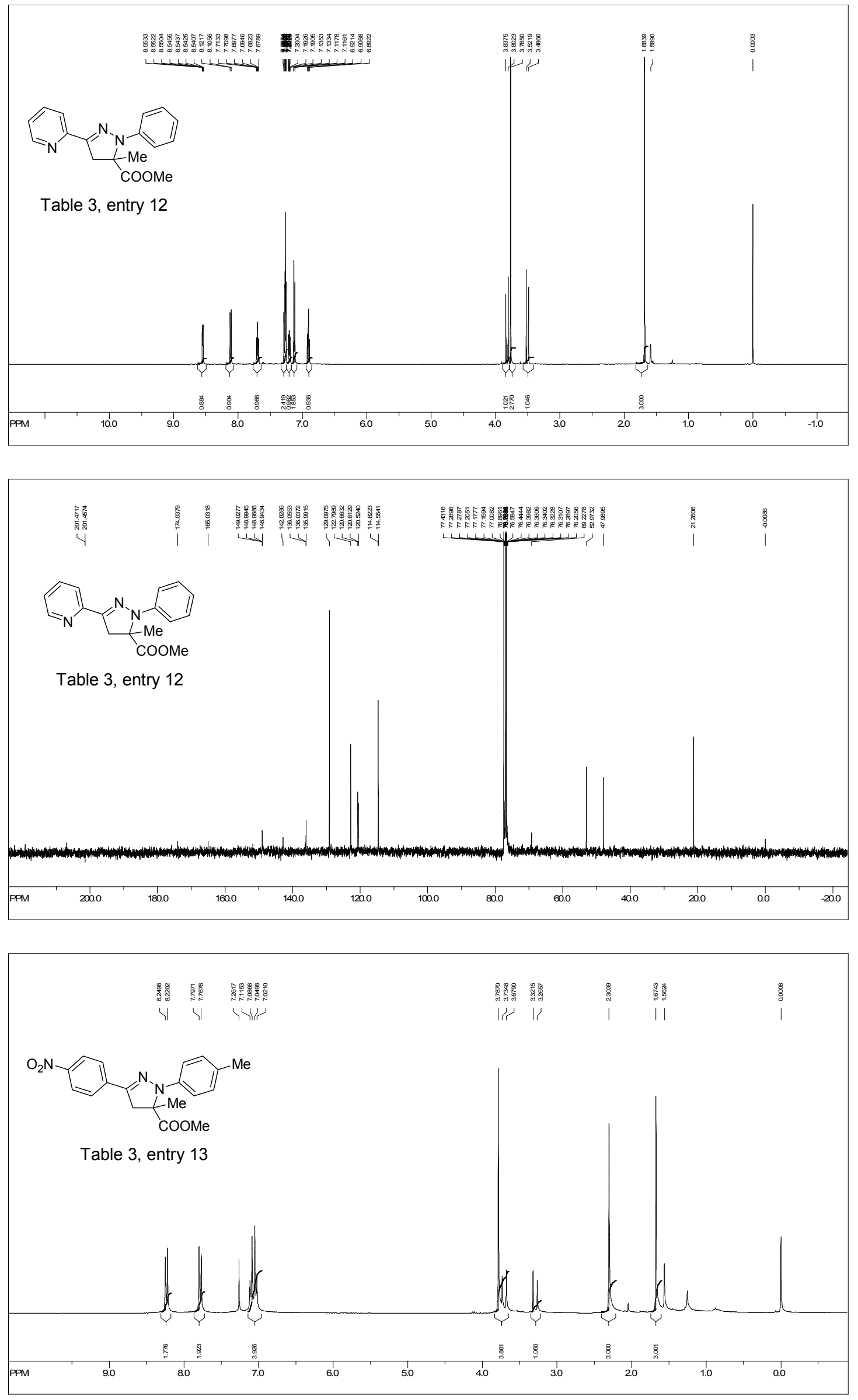

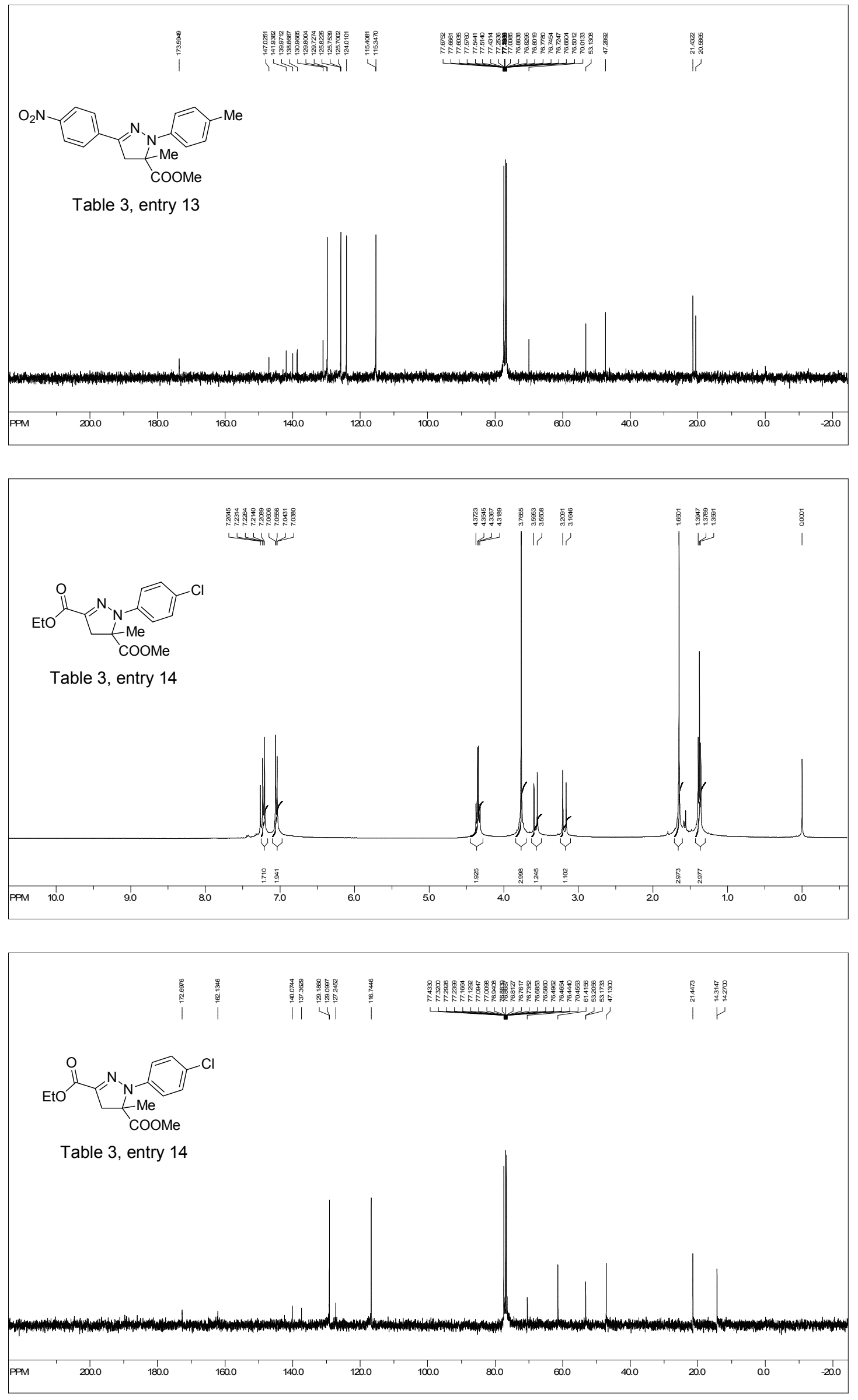

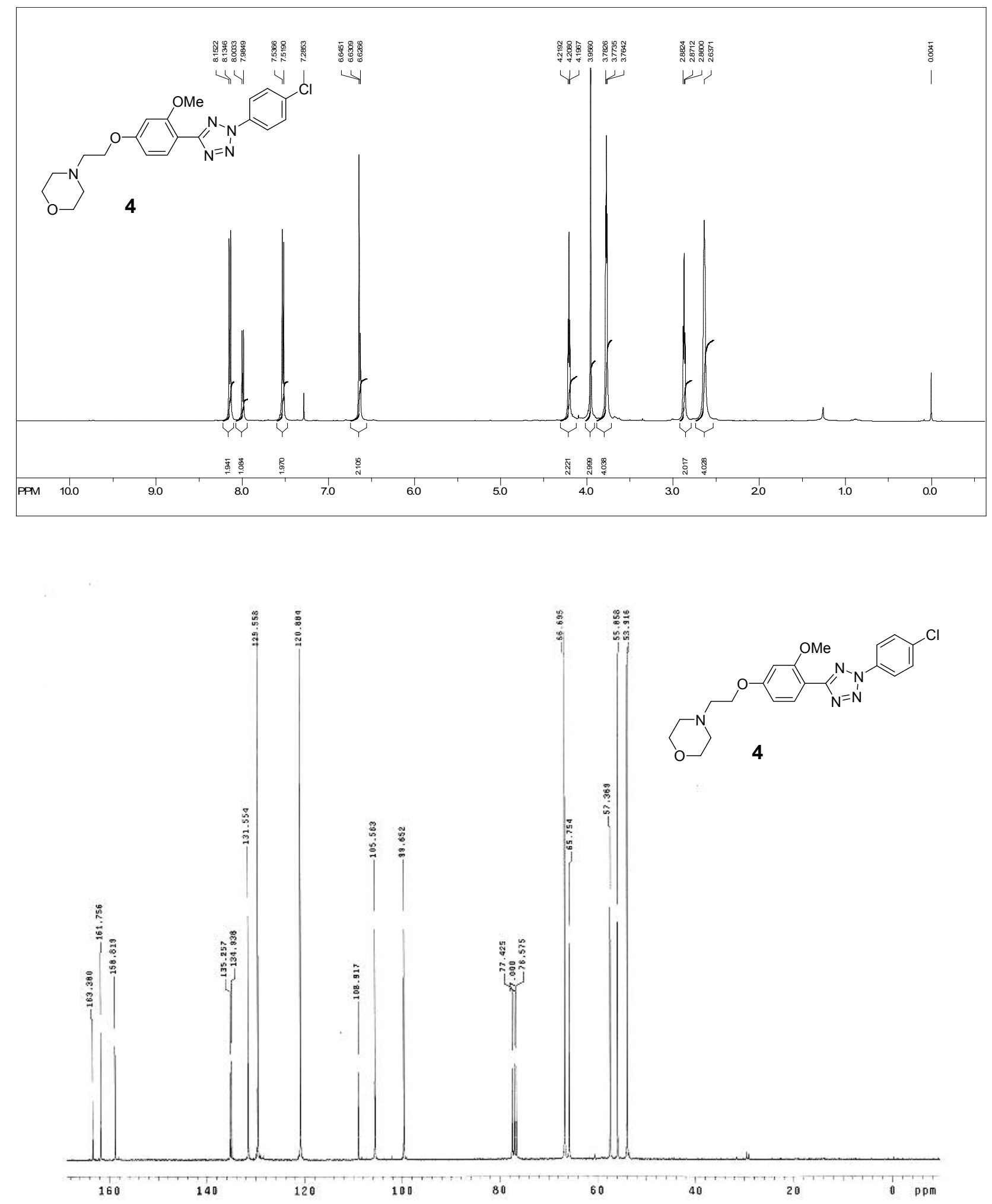

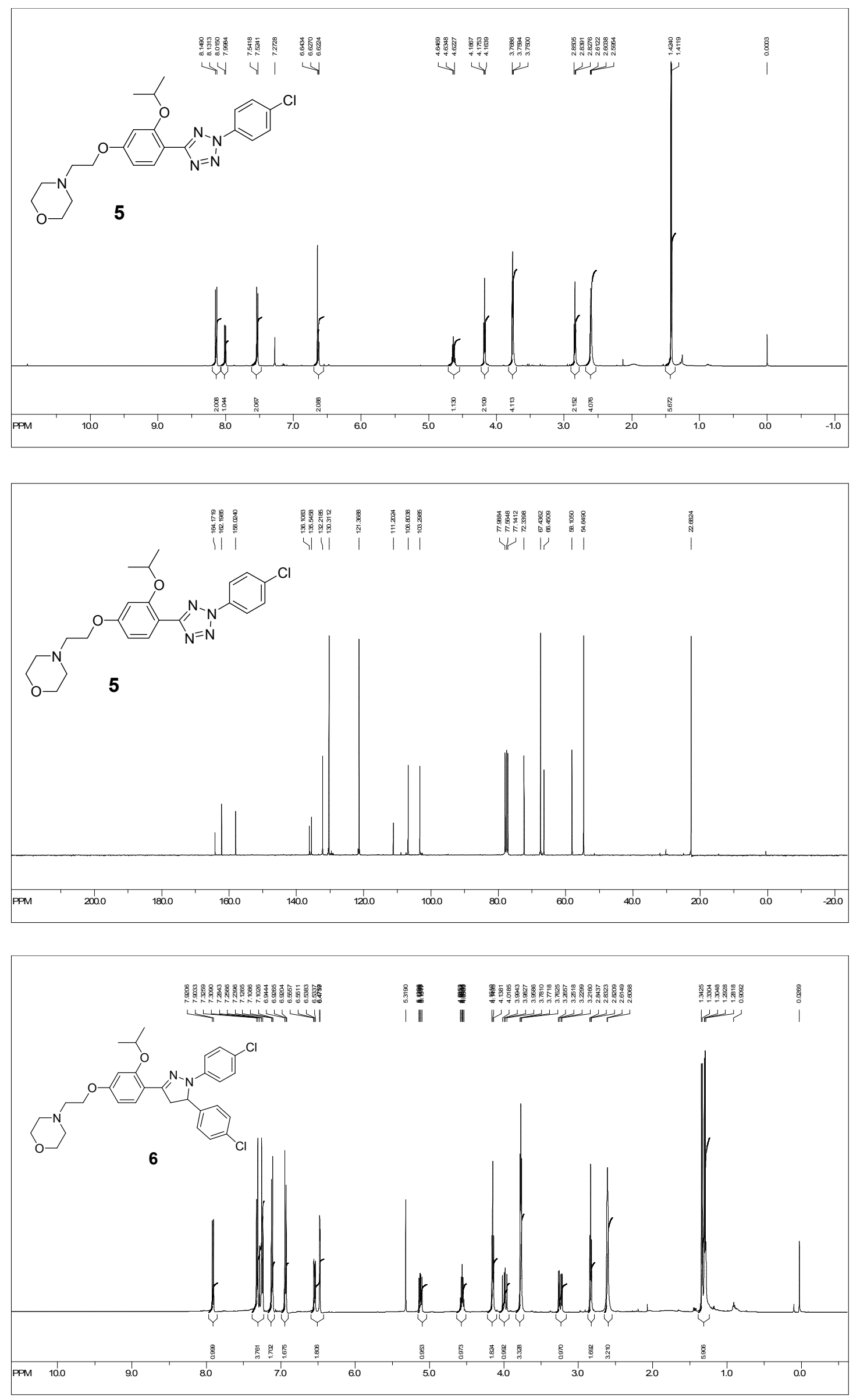

30 


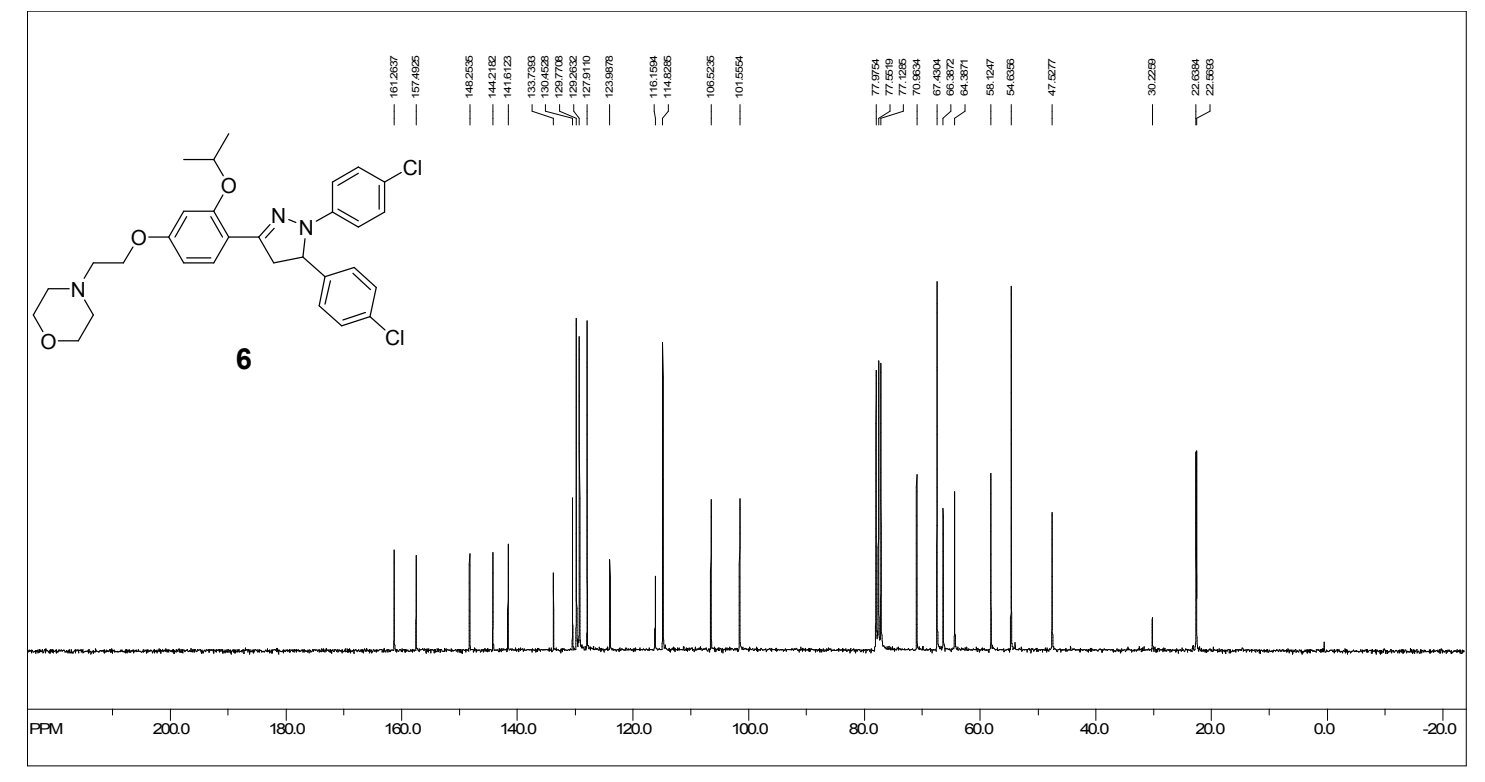

